\title{
Focal-plane and aperture-plane heterodyne array receivers for millimeter-wave radioastronomy-A comparison
}

Article in International Journal of Infrared and Millimeter Waves · August 1988

DOI: $10.1007 / \mathrm{BF} 01026393$

CITATIONS

14

2 authors:

John Anthony Murphy

National University of Ireland, Maynooth

376 PUBLICATIONS 17,308 CITATIONS

SEE PROFILE
READS

71

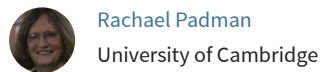

96 PUBLICATIONS 1,355 CITATIONS

SEE PROFILE

Some of the authors of this publication are also working on these related projects:

Herschel-HIFI View project 


\title{
FOCAL-PLANE AND APERTURE-PLANE HETERODYNE ARRAY RECEIVERS FOR MILLIMETER-WAVE RADIOASTRONOMY - A COMPARISON
}

\author{
J. Anthony Murphy \\ Experimental Physics Department \\ Maynooth College \\ County Kildare, Ireland \\ and \\ Rachael Padman \\ Mullard Radio Astronomy Observatory \\ Cavendish Laboratory \\ Cambridge CB3 OHE UK
}

Received April 24, 1988

Abstract: In this paper we consider the maximization of the throughput of a single large antenna, for two possible array configurations: focal plane imaging arrays and aperture plane phased arrays. We discuss trade-offs between the two types of array in terms of field of view, sampling efficiency and time to map a source. We also discuss limits on the number of feed elements in an imaging array imposed by the deterioration in aperture efficiency off-axis.

Key Words: Arrays, millimeter telescopes, heterodyne receivers. 


\section{Introduction.}

Coherent detectors, such as heterodyne receivers, are limited in response to a single spatial mode. In infrared terminology, the etendue or throughput $(A \Omega)$ can never be greater than $\approx \lambda^{2}$. Thus, unlike the situation in incoherent infrared astronomy, the throughput of the telescope cannot be increased merely by increasing A (for example, by opening a diaphragm in the focal plane). Array receivers, however, offer the possibility of a significant increase in the efficiency (or throughput) of single dish radiotelescopes. At the shorter wavelengths typical of the current generation of millimeter-wave telescopes the field of view is extensive, and the possible increase of throughput correspondingly large.

The basic theory of array receiving systems is well known, and has been developed at some length for interferometric and phased arays of independent antennas (see e.g. refs.[1-5] and references therein). However, the question of how best to maximize the throughput of a single large antenna has not received the same attention, although there have been a number of recent papers and articles dealing particularly with antenna structures suitable for millimeter and submillimeter wavelength arrays [6-8]. In this paper we explore the trade-offs between sampling, efficiency (of an individual element) and field of view, using a consistent notation which facilitates the comparison of two fundamental array types.

The possible configurations for an array receiver are bounded by two extremes. These are the focal plane array, where the array of detectors is placed on the telescope focal plane (see fig.1), and the aperture plane array, where the array is placed at a conveniently sized image of the telescope aperture, (e.g. as in fig. 1). In section II we consider how each array reconstructs a map of the brightness distribution of the source. Questions of how many array elements are needed and how long it takes to map a field of view are addressed in section III. We assume a simple source and telescope configuration as examples so that quantitative comparisons can be made. In section IV we derive a relationship for the deterioration of the aperture efficiency of an off-axis imaging array element. 


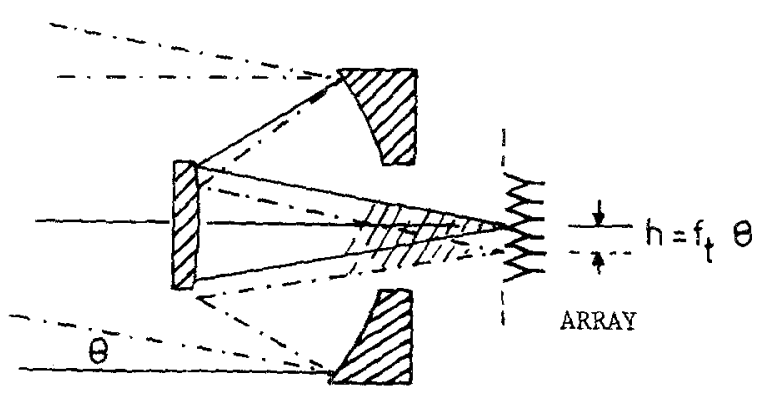

FOCAL PLANE ARRAY

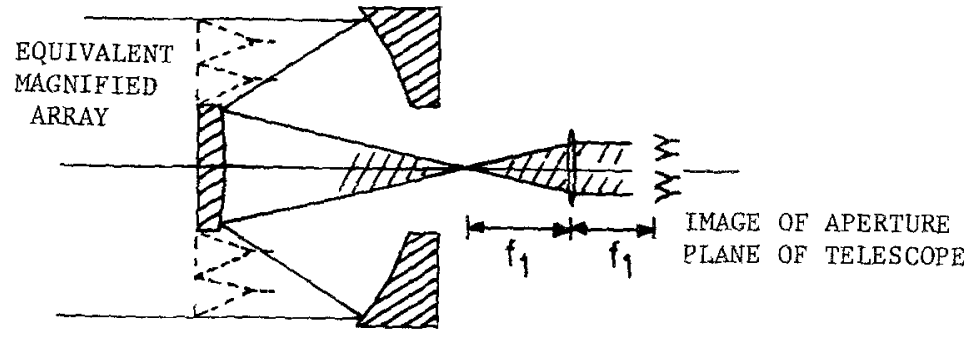

APERTURE PLANE ARRAY

FIG. I SCHEMATIC OUTLINE OF A FOCAL PLANE AND APERTURE PLANE ARRAY 


\section{Types of arrays}

In this section it is assumed that the individual mixer elements have aperture feeds, such as the rectangular horn. The theory of mapping a field with the two types of array is considered.

\section{Focal plane arrays.}

Let us examine the case when an array of homs is placed on a part of telescope focal plane where there is an undistorted diffraction limited image of the sky. In Appendix 2 it is shown that, for hom $i$ with power pattern on the sky $\mathrm{G}\left(\boldsymbol{\theta}-\boldsymbol{\theta}_{1}\right)$, the power coupling to a celestial source with the source brightness distribution $\mathrm{B}(\theta)$, i.e.:

$$
P_{i}=\int B(\theta) G\left(\theta_{i}-\theta\right) d A_{\theta}
$$

Note: The (2-component) vector $\boldsymbol{\theta}$ is such that the components of $\theta$ correspond to the projection onto two orthogonal axes in the aperture plane of a unit vector in the source direction (i.e. correspond to the direction cosines for the two axes). The notation $\int \mathrm{dA}_{\theta}$ indicates the double integral over the two components of $d \theta$ (e.g. $\int \mathrm{dA}_{\theta}=\iint \mathrm{d} \theta_{\mathrm{x}} \mathrm{d} \theta_{\mathrm{y}}$ ).

A point $\mathbf{r}$ in the focal plane is related to a particular direction $\theta$ by $\boldsymbol{\theta}=-\mathbf{r}$, where $\mathbf{r}$ is a dimensionless quantity equal to the distance from the axes divided by $f_{t}$. The power patterns $G=|h(-\theta) * w(-\theta)|^{2}$, where $h$ is the hom-"phase centre" field and $w$ is the fourier transform of the telescope aperture weighting function $\mathrm{W}$ which represents the truncation of the incident beam at the telescope aperture.

The map $\hat{B}(\theta)$ consists of a set of samples corresponding to the centres of the horns in the focal plane (or equivalently corresponding to the set of directions of the array of beams on the sky): $\hat{B}\left(\theta_{i}\right)=P_{i}$. Thus, we can write:

$$
\hat{\mathrm{B}}\left(\boldsymbol{\theta}_{\mathrm{i}}\right)=\mathrm{B}\left(\boldsymbol{\theta}_{\mathrm{i}}\right) *\left\{\left.\operatorname{lh}\left(-\boldsymbol{\theta}_{\mathrm{i}}\right)^{*} \mathrm{w}\left(-\boldsymbol{\theta}_{\mathrm{i}}\right)\right|^{2}\right\}
$$

The focal plane intensity distribution $\hat{B}(\theta)$ is bandlimited because of the finite spatial extent of the telescope as shown in (2), and can therefore be reconstructed perfectly if sampled at a sufficiently small spacing. This critical spacing (the Nyquist rate) is determined by the resolution of the telescope, given by $G(\theta)$. For the optical transfer function [8] corresponding to a circular aperture with uniform illumination the Nyquist sampling interval on the focal plane is $\frac{1}{2} \lambda F$ where $F$ is the geometric focal ratio, $f_{t} / D$, ( $D$ being the diameter of the telescope aperture). That is, we require a beam spacing on the sky of $\frac{1}{2} \lambda / \mathrm{D}$. The sampling interval is equal to $41 \%$ of the radius of the first null of the Airy pattern produced in the focal plane by a point source at infinity. 


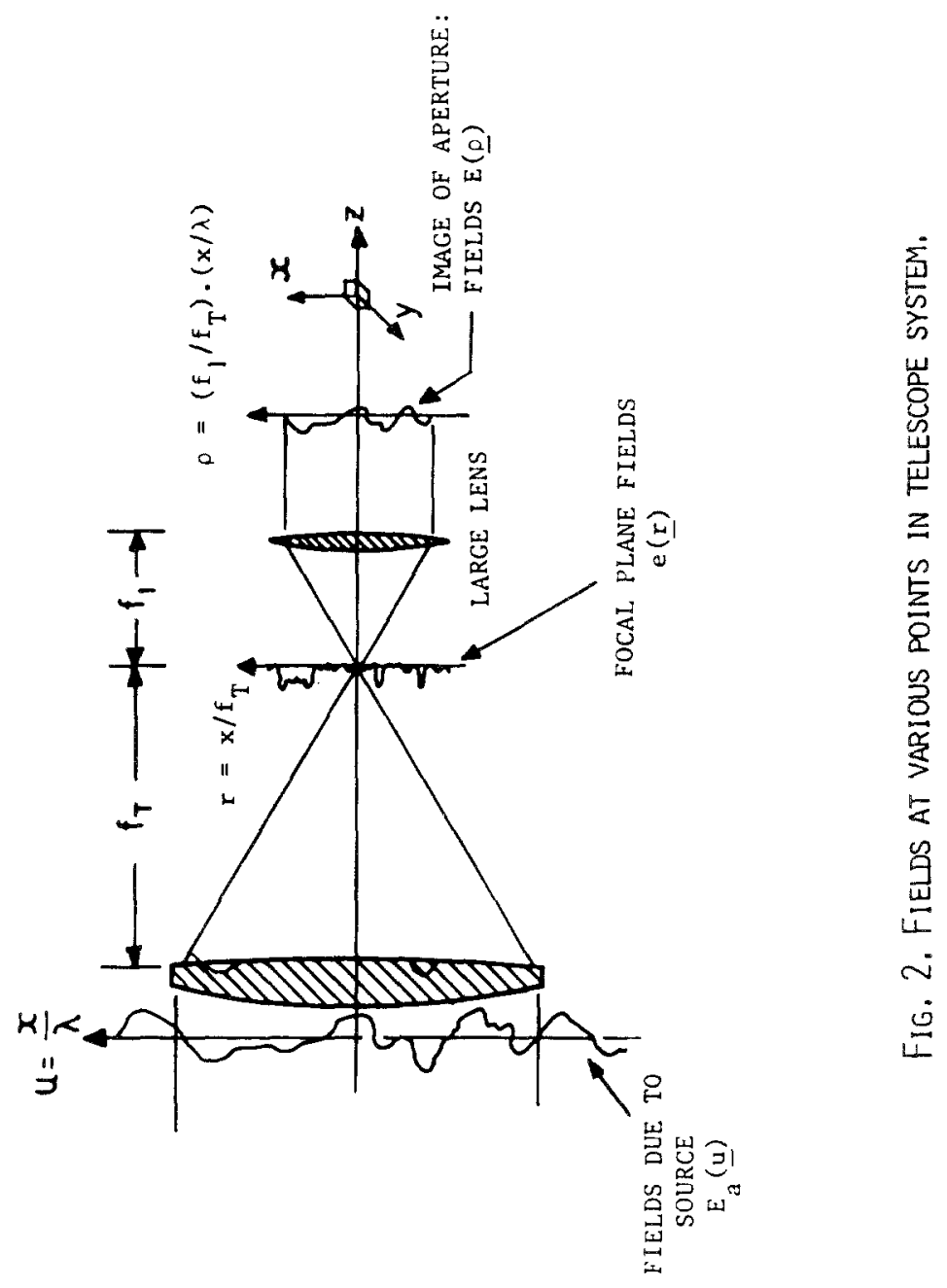


Reference is sometimes made to the possibility of measuring higher spatial frequencies by measuring the voltage rather than intensity [6], the argument being that the process of (square-law) detection effectively doubles the spatial frequencies in the image. In the radioastronomical case we do not usually expect any coherence between the image points separated by more than a beamwidth and even that is enforced only by the truncation at the edge of the aperture. In other words, relative to some arbitrary reference position the fields at any point in the focal plane time-average to zero, and we are interested only in discovering the structure in the intensity pattern approximating the brightness temperature distribution of the true sky, for which the usual sampling criteria apply.

\section{Aperture plane arrays}

The fields at the image of the aperture plane are a scaled version of the truncated aperture field distribution $E_{a}$, i.e., $E(\rho)=E_{a}(\gamma \rho) W(\gamma \rho)$ where $\rho$ is a point on the image aperture plane and $\gamma=-f_{1} / f_{t}$. (see fig.2). We can therefore choose to work in either the image plane or the actual aperture plane by considering the image fields or the image horns respectively. The following treatment assumes that we are working in the aperture plane itself (i.e. considering an image array at the true telescope aperture plane).

There are two basic ways in which an array could be configured:

(i) as a simple phased array, where a series of phase gradients is applied across the array, all the outputs for a particular gradient being added together to produce a series of beams on the sky [4]; see fig.3.

(ii)as a synthesis array where the elements of the array are connected in interferometric pairs to sample the complex correlation function of the aperture fields[5].

\section{(i) Phased array}

For a phased array we define the phase gradient $\theta_{1}=\left(\theta_{x}, \theta_{y}\right)$ to be the phase increment per unit wavelength divided by $2 \pi$ along the two orthogonal axes of the array. We show in Appendix 2 that the total power output $\mathrm{P}\left(\boldsymbol{\theta}_{1}\right)$ from such an array is given by:

$$
P\left(\theta_{i}\right)=\int d_{A} B(\theta)|H(\theta)|^{2} \cdot\left|G\left(\theta-\theta_{i}\right)\right|^{2}
$$

$\mathrm{G}(\boldsymbol{\theta})$ can be written as a convolution of $\mathrm{U}(\boldsymbol{\theta})$ (the Fourier transform of a 2-dimensional array of $\delta$-functions) and $w(\theta)$ (the Fourier transform of $\mathrm{W}(\boldsymbol{\theta})$, the telescope aperture weighting function). $U(\boldsymbol{\theta})$ has the form of the far field of an infinite phased array of isotropic radiators. 


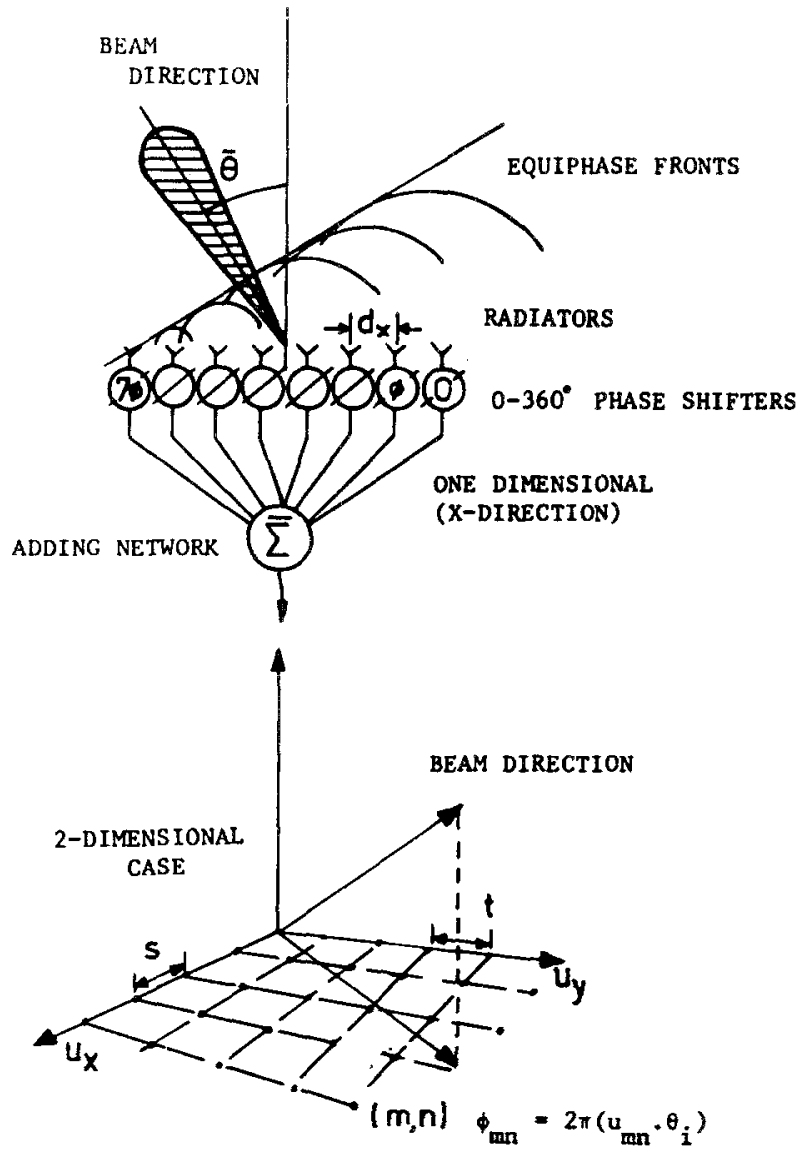

Fig. 3 Beam Steering using phase Shifters at EACH RADIATING POINT, 
The map $\hat{B}$ consists of a set of samples where $\hat{B}\left(\theta_{1}\right)=P\left(\theta_{1}\right)$. :

$$
\hat{\mathrm{B}}\left(\boldsymbol{\theta}_{\mathrm{i}}\right)=\mathrm{B}\left(\theta_{\mathrm{i}}\right) \cdot\left|\mathrm{H}\left(\boldsymbol{\theta}_{\mathrm{i}}\right)\right|^{2 *}\left\{\left|\mathrm{U}\left(\boldsymbol{\theta}_{\mathrm{i}}\right) *_{\mathrm{W}}\left(\boldsymbol{\theta}_{\mathrm{i}}\right)\right|^{2}\right\}
$$

It is possible to fill in all the spacings and map a source without moving the telescope, provided the elements are connected to a series of constant slope phase tapers corresponding to all the necessary spacings.

The resolution of the array is determined by the spatial extent of $W$ through $\mathrm{w}(\theta)$, the intrinsic telescope beam, while the sampling in the aperture plane determines the distance between gratin responses through $\mathrm{U}(\boldsymbol{\theta})$. As in the focal plane imaging receiver the observed $\mathrm{P}_{\mathrm{i}}\left(\theta_{\mathrm{i}}\right)$ contain spatial frequencies up to the resolution of the telescope and the Nyquist interval is $\lambda / 2 \mathrm{D}$.

\section{(ii) Synthesis array.}

In this case the outputs from the detectors are connected in pairs (by adding and square-law detecting the output or, alternatively, by multiplying and low pass filtering the output). We define the baseline $b_{\text {. }}$ to be the spacing (in the aperture plane) of the images of the two elements of the interferometer (which is physically located at the image of the aperture plane as in fig.1). In appendix 2 we show that the map $\hat{\mathrm{B}}(\boldsymbol{\theta})$ is given by:

$$
\begin{aligned}
& \hat{\mathrm{B}}(\boldsymbol{\theta})=\int \mathrm{dA}_{\theta}, \mathrm{B}\left(\boldsymbol{\theta}^{\prime}\right)\left|\mathrm{H}\left(\theta^{\prime}\right)\right|^{2} \sum_{\mathrm{i}}\left\{\mathrm{W}_{\mathrm{b}}\left(\mathrm{b}_{\mathrm{i}}\right) \cdot \cos \left[2 \pi \mathrm{b}_{\mathrm{i}} \cdot\left(\boldsymbol{\theta}-\boldsymbol{\theta}^{\prime}\right)\right]\right\} \\
& \hat{\mathrm{B}}(\boldsymbol{\theta})=\left\{\mathrm{B}(\boldsymbol{\theta})|\mathrm{H}(\theta)|^{2}\right\}^{*}\left\{\Sigma_{\mathbf{i}} \mathrm{W}_{\mathrm{b}}\left(\mathrm{b}_{\mathrm{i}}\right) \cdot \cos \left[2 \pi \mathrm{b}_{\mathbf{i}} \cdot \theta\right]\right\} \text {. }
\end{aligned}
$$

That is, the true source distribution $\mathbf{B}(\theta)$ has been multiplied by the primary beam power pattern $\left|\mathrm{H}_{\mathrm{i}}(\boldsymbol{\theta})\right|^{2}$ and convolved with the synthesised beam. We can rewrite this in terms of the array-plane quantity $\rho$ and the magnification factor $\gamma=-f_{1} / f_{t}$ as:

$\hat{\mathbf{B}}(\boldsymbol{\theta})=\left\{B(\theta)|\mathrm{H}(\theta)|^{2}\right\}^{*}\left\{\Sigma_{\mathrm{i}} W\left(\gamma \rho_{\mathrm{i}_{1}}\right) \cdot W\left(\gamma \rho_{\mathrm{i}_{2}}\right) \cdot \cos \left[2 \pi\left(\rho_{\mathrm{i}_{1}}-\rho_{\mathrm{i}_{2}}\right) \cdot \boldsymbol{\theta}\right]\right\}$

Note that the synthesised beam is symmetric, but unlike the beam of a phased array it is not constrained to be positive and in fact contains zero power total power.

A synthesis array is equivalent to a phased array except that the beam doesnot contain a d.c. term, the beam produced by the two configurations are otherwise equivalent and in the remainder of this discussion we just consider the phased array. 


\section{Time to map a source.}

We wish to compare the merits of the two types of arrays when it comes to the question of the number of elements and the length of time it takes to map a source.

In a focal plane array with elements dimensioned so as to maximise the aperture efficiency the fields at a hom aperture couple well to the Airy diffraction spot produced on the focal plane by a point source. Nyquist sampling, however, implies inter-hom spacing of $0.41 \times$ radius of the Airy disc, constraining the size of individual horns to be less than that required for good coupling. If high efficiency is desired then the telescope must perforce be moved to record the missing samples - we refer to this technique as hybrid mapping.

It is clearly always impossible to achieve full (Nyquist) sampling and maximum aperture efficiency simultaneously. A fundamental limit can be found simply from conservation of energy: if an array element having a circularly symmetric beam pattern and aperture efficiency of $\eta_{a}$ is surrounded by $\mathrm{N}$ equidistant similar elements, then the maximum response of any one of the $\mathrm{N}$ elements to a point source is just $\left(1-\eta_{\mathrm{a}}\right) / \mathrm{N}$, which yields an upper limit to the coupling of one of these elements to the source of $1 / \mathrm{N}$, regardless of $\eta_{\mathrm{a}}$ ! It is obviously possible to pack structures such as planar, slot, finline or bow-tie antennas closer together than is suggested by the fundamental limit, but in that case the mutual impedances of all antennas will conspire to reduce the response of all elements to satisfy the above constraint. Results such as those reported in [8] can be understood if it is recognised that under the test condition the adjacent antennas were not terminated, and hence presumably presented a large mismatch to the incoming radiation. Later we examine the throughput of a close-packed focal plane array of rectangular hom antennas where the size of the horns, and hence $\eta_{a}$, is allowed to vary.

By contrast, an array of horn antennas in an aperture plane configuration has the advantage that there need be no missing spacings (as in the focal plane array) so a map can be made immediately. The drawback, however, is that the map will contain the grating responses of the array at $\pm \lambda / \mathrm{d}$ ( $\mathrm{d}$ is the spacing between the image horns at the aperture), which will tend to cause confusion for sources that have extent greater than about $\lambda / \mathrm{d}$. Thus, there is a limitation on the field of view of this type of array. Also, the signal to noise in the case of an aperture plane array is degraded according to the ratio of the power in the main lobe to the total power in the pattern. This reduction in sensitivity is severe for beams at the edge of the field of view of the array. 
When comparing the performance of the two types of arrays for mapping sources all these factors have to be considered. In order to illustrate the various contributions of these factors let us suppose for simplicity that we have:

a square source (of side angular extent $\theta_{\mathrm{S}}$ )

a square aperture (of side length D)

a close packed array of long rectangular horns with dimensions $\mathrm{a} \times \mathrm{b}($ E-plane $\times \mathrm{H}$-plane $)$.

We also assume that a $\mathrm{TE}_{10}$ field distribution $\mathbf{E}_{\mathrm{h}}$ exists at the horn mouths. As the homs are long we assume the phase error across the horn apertures is negligible. The far field pattern $\mathrm{H}(\boldsymbol{\theta})$ of a single hom a distance $R$ from the horn aperture[9] is then given by:

$$
H(\theta)=-\frac{1}{2} \pi C \frac{\cos X}{\left[X^{2}-\left(\frac{1}{2} \pi\right)^{2}\right]}-\frac{\sin Y}{Y}
$$

whe re

$$
\begin{aligned}
& C=j a b / \lambda . E_{0} / R \cdot \exp (-j k R) \\
& X=\pi a / \lambda \cdot \theta_{X} \text { and } \quad Y=\pi b / \lambda \cdot \theta_{y}
\end{aligned}
$$

\section{(a) Coupling to source.}

In Appendix 3.1 we show that for a focal plane array the forward gain of the telescope is maximised when the horn dimensions are given by $\mathrm{a}=1.88 \lambda \mathrm{F}$ and $\mathrm{b}=1.36 \lambda \mathrm{F}$, giving an aperture efficiency of 0.745 . The Nyquist sampling rate is $\frac{1}{2} \mathrm{~F} \lambda$, so that the undersampling factors are 2.72 along the $y$-axis (E-plane) and 3.76 along the $x$-axis (H-plane) giving an overall undersampling factor of 10.2. We separately calculate the spillover efficiency, as being that fraction of the hom power pattern which does not intercept the telescope aperture (this quantity is also sometimes known loosely as beam efficiency). In Appendix 3.1 we also show that the total spillover efficiency is $\eta_{\mathrm{S}}=0.831$.

The forward coupling of an aperture plane array is reduced by two mechanisms. First, some fraction of the total power pattern is contained in the secondary "grating lobes", and is assumed to be wasted. The fraction lost to grating lobes depends on the exact position of the "synthesised beam" within the primary beam pattem defined by the individual array elements (see fig.4). In Appendix 3.2 we show that the maximum value for the fraction of the power in the main beam occurs when the synthesised beam is coincident with the centre of the primary power pattern (i.e. for zero phase taper), and has a value: $\eta_{b m a x}=$ $8 / \pi^{2}=0.8106$. At the edge of the field of view, $\eta_{b}$ takes on its minimum value as the response of the primary beam decreases away from the centre of the source. This is given by $\eta_{b \min }=0.2026$ (see Appendix 3.2). 


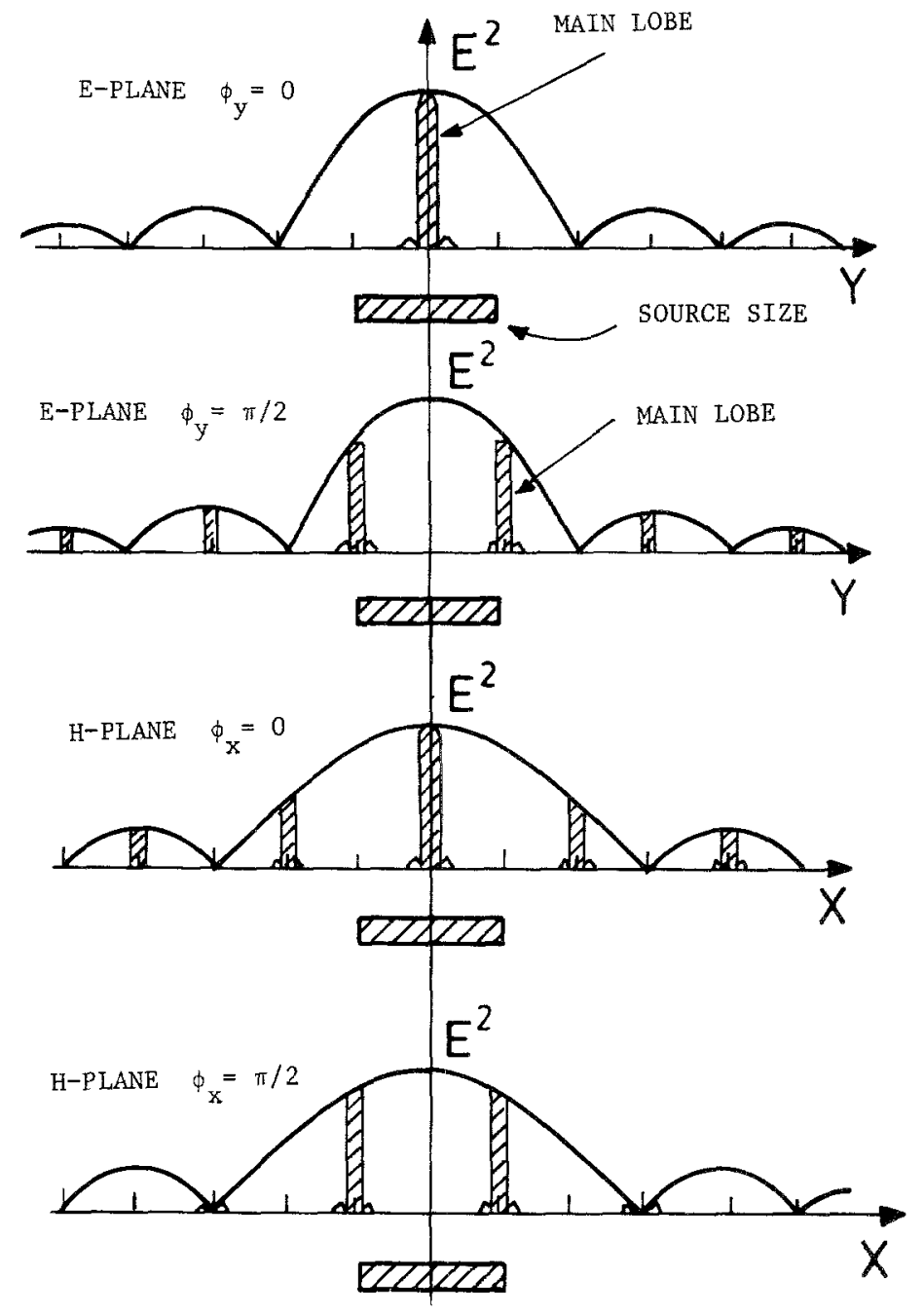

FIG, $4 E^{2}\left(\phi_{X}, \phi_{Y}\right)$. AS A FUnCTION OF $(X, Y)$. 
The number of horns $\mathrm{n}_{\mathrm{f}}$ required in a focal plane array just to cover the area of the source is given by the area of the image divided by the area of an individual horn aperture - we show in Appendix 3.1 that this has a value $\mathrm{n}_{\mathrm{f}}=(1 / 2.55) \cdot \theta_{\xi} /(\lambda / \mathrm{D})^{2}$. For an aperture plane array the number of horns $n_{a}$ is set by the requirement to avoid aliasing, and so the phase taper must amount to less than $\pi$ radians per element. It is shown in Appendix 3.2 that we therefore require at least $\mathrm{n}_{\mathrm{a}}=$ $\theta_{s} /(\lambda / D)^{2}$ homs to yield an unconfused field of view of angular dimension $\theta_{\mathrm{S}}$.

(b) Total time to map a source.

We now wish to compare the time it takes to map a source to a given signal to noise using both types of array.

We denote antenna temperature and source temperature by $T_{a}$ and $T_{B}$, respectively. We are interested in the change $\Delta T_{B}$ over a solid angle occupied by the "main beam" on source. The corresponding change in antenna temperature $\Delta \mathrm{T}_{\mathrm{a}}$ is given by: $\Delta \mathrm{T}_{\mathrm{a}}=1 / \lambda^{2} \cdot \Delta \mathrm{T}_{\mathrm{B}} \cdot \mathrm{d}(\boldsymbol{\theta}) \mathrm{d} \mathrm{A}_{\theta}$, where $A(\theta)$ is the effective area of the telescope in direction $\theta$ and the integral is over the main beam plus close-in side lobes. Beam efficiency $\eta_{B}$ is defined to be $1 / \lambda^{2} \int_{\text {main beam }} A(\theta) d A_{\theta}$. If there are no sidelobes $\eta_{\mathrm{B}}=1$, (assuming no ohmic losses). Thus, $\Delta \mathrm{T}_{\mathrm{a}}=$ $\eta_{\mathrm{B}} \Delta \mathrm{T}_{\mathrm{B}}$. The minimum change in source temperature $\left(\Delta \mathrm{T}_{\mathrm{B}}\right)_{\min }$ that is detectable with a system with a system noise temperature $\mathrm{T}_{\text {sys }}$ is given by $[10]$ :

$$
\left(\Delta \mathrm{T}_{\mathrm{B}}\right)_{\min }=2 \mathrm{~T}_{\mathrm{sys}} / \eta_{\mathrm{t}} \eta_{\mathrm{B}} \sim \mathrm{t}
$$

where $v$ is the bandwidth of the receiver and $t$ is the total observing time.

In the case of a focal plane array $\eta_{\mathrm{B}}=$ spillover efficiency $\left(\eta_{\mathrm{S}}\right)$ as this corresponds to power lost into far out side lobes. In the case of an aperture plane array $\eta_{\mathrm{B}}=$ fraction of power in the main lobe for a particular beam.

Thus, denoting by $t$ the time required to map the source using a minimum number of homs to a given $S / N$ (and including undersampling factors), the ratio of the times for the two arrays is given by:

$$
\begin{aligned}
\mathrm{t}_{\mathrm{a}} / \mathrm{t}_{\mathrm{f}} & =\left(1 / \eta_{\text {bmin }}{ }^{2}\right) /\left(\text { undersampling factor } / \eta_{\mathrm{s}}{ }^{2}\right) \\
& =(1 / .2026)^{2} /\left(4 \times 2.55 /(0.831)^{2}\right) \\
& =1.647
\end{aligned}
$$

i.e the focal plane array is faster by a factor $\approx 1.6$. 


\section{(c) Alternative schemes.}

The rather surprising result that a focal plane array maps more quickly than an aperture plane array is due mostly to the rather large value for $1 / \eta_{\mathrm{bmin}}$ for the aperture plane array. The sensitivity of the beams at the edge of the field is low. For a source extended over a large number of pixels, such that the number of pixels is greater than the sampling factor, there are a number of ways to improve the speed of the aperture plane array. The most straightfoward way to give all pixels equal sensitivities for a large source is to move the telescope in 1-pixel increments, so that ultimately all pixels receive the same effective value for $\Delta \mathrm{T}_{\mathrm{B}}$. Alternatively, we could instead just map a fraction of the source using only the centre beams with higher sensitivity and then move the telescope to complete mapping of the source (i.e. we "undersample" in the sense that we do not map the whole source at once as in our earlier calculations).

Equally, however, it turns out that we can also map the source faster with the focal plane array if we allow a closer spacing in the focal plane with a higher spillover loss, and assume that the number of horns is increased to cover the source field of view (essentially we are trying to maximize the throughput per unit area of focal plane). Clearly, if the number of horns is fixed the observing time is maximised when $\eta_{a}$ is optimised, provided the field of view is less than or equal to the size of the source. The time it would take to map the source relative to the maximum efficiency case is given from (3.7) by: $t \propto U_{\mathrm{f}} / \eta_{\mathrm{s}}{ }^{2}$, where $\eta_{\mathrm{S}}$ is the spill-over loss (as discussed in section 3.1 (a)) and $U_{f}$ is the undersampling factor: $U_{f}=4 a b / F^{2} \lambda^{2}$.

We show in Appendix 3.1 that the minimum mapping time is achieved for homs having dimensions $a=1.305 \mathrm{~F} \lambda$ and $\mathrm{b}=0.96 \mathrm{~F} \lambda$ (corresponding to an undersampling factor of 5.0), when the aperture efficiency decreases to 0.820 times its maximum value. This minimum time is 0.720 times that using the maximum aperture efficiency criterion.

Applying a similar calculation to the case of an aperture plane array, in Appendix 3.2 we show that the time is minimised when $p$, the fraction of the total possible number of beams which could have been used to map the source, is 0.605 . This yields a reduction in the time to map the source by a factor of 2.2 (thus a map can be made to the same signal to noise over 2.2 times as fast as if we map with all the beams). When we impose the constraint that $p$ must be a sub-multiple of 1 , then we find $p=0.5$, the reduction factor is still 2.1 ; still a saving of over $50 \%$ on the time required to make the map. 
Note, however, that if the unconfused field of view of the array is now less than twice the size of the source then any "hybrid" technique (rastering in 1-pixel increments or greater) will yield a confused image which will require deconvolution (using, for example, CLEAN or Maximum Entropy).

\section{Field of View of a Cassegrain Telescope.}

An important limitation on the size of a focal plane array will be the deterioration in the quality of the field of view as the feed is moved off axis. A Cassegrain telescope (where the primary is paraboloidal and the subreflector hyperboloidal) is free from spherical aberration, but does suffer from off-axis aberrations viz. coma, astigmatism and field curvature. In microwave and millimetre-wave antennas we are interested primarily in the aperture efficiency, i.e. the coupling between the image of a point source in the field of view and a microwave feed on the telescope focal plane. Aberrations cause a deterioration in the aperture efficiency as one moves away from the telescope optical axis.

It is usually assumed that the microwave Cassegrain behaves like a single paraboloidal dish (known as the "equivalent paraboloid") with a focal length equal to the effective focal length of the Cassegrain system, and with a diameter the same as that of the primary[11],[12]. The results of Ruze [13] for the effect of displacing a feed in a single paraboloidal dish are then sometimes applied. However, Ruze considered only the case where the displaced feed points at the centre of the parabolic reflector and is placed on the Petzval surface. Also, for high F-ratio systems, Ruze's results can only be applied for very small displacements[14]. Thus, these results from the microwave literature are not applicable to a flat focal plane array on a high $F$ ratio Cassegrain system. To summarize there are basically two reasons why the usual microwave approach is inadequate:

(i) on high F-ratio systems coma is not the dominant aberration present (as is the case in Ruze, for example).

(ii) if one is dealing with a flat array on the focal plane without a field lens then non-symmetrical illumination of the subreflector by the off-axis feeds leads to more severe spill-over effects than for an on-axis feed.

In our study of array systems we can however make use of knowledge gained from traditional geometrical optics studies, since diffraction effects can usually be neglected outside the telescope focal region. It is useful to consider the quantity used in optical systems called the "aberration function"[15]. In a general image forming system shown in 
fig.5 a paraxial image of an object at $\mathrm{P}$ a distance 1 off-axis is formed at $\mathrm{P}^{\prime}$ with no distortion effects a distance $h$ off-axis. The aberration function is defined by [15]:

$\Phi(Q)=$ optical path length (PQP') - optical path length (POP')

where $O$ is the centre and $Q$ is any point on the exit pupil of the optical system. For a Cassegrain telescope the exit pupil is defined by the subreflector (fig.6). In Appendix 4 it is shown that for a telescope where the focal length of the primary reflector $f_{p}$ is much less than the effective focal length $f$, curvature of field is the dominant aberration for values of $h>\left(f_{p} / f\right)$ a. In that case $\Phi$ can be written as a function of the radial coordinate $\mathrm{I}$ for point $\mathrm{Q}$ as:

$$
\Phi(\mathrm{r}) \approx \gamma\left(\mathrm{h} / 32 \mathrm{~F}^{3}\right)(\mathrm{h} / \mathrm{a})(\mathrm{r} / \mathrm{a})^{2} \quad \text { where } \gamma=\mathrm{f} / \mathrm{f}_{\mathrm{p}}
$$

The plane wave incident on the primary mirror from a distant point source will be focused to a distorted and truncated spherical wave impinging on $\mathrm{P}$ '. The phase deviation at the subreflector is given by $\mathrm{k} \Phi(\mathrm{Q})$. The distorted wave after reflection from the subreflector is given by:

$$
\begin{aligned}
E_{S} & =\exp \left(+j k\left[r^{2} / 2 d_{S}+\Phi(r)\right]\right) \\
& \approx \exp \left(+j k\left[r^{2} / 2 d_{S}+\gamma\left(h / 32 F^{3}\right)(h / a)(r / a)^{2}\right]\right)
\end{aligned}
$$

where $d_{S}$ is the subreflector-telescope focal plane distance.

The radiation field of a microwave feed placed on the telescope focal plane will have spherical phase fronts at the exit pupil (the subreflector), provided the subreflector is in the far field of the feed. Let us suppose that a microwave feed offset by $h$ laterally from the focal point produces a Gaussian beam in its far field. If we choose to have a flat array, then the beam propagates parallel to the axis of the telescope and the centre of the beam at the subreflector is displaced by h. The electric field of the feed beam at the subreflector (exit pupil; see fig.5) can be written :

$$
\mathrm{E}_{\mathrm{g}}(\mathrm{r}, \varphi) \propto \exp \left(-\left[(\mathrm{r} \cos \varphi-h)^{2}+\mathrm{r}^{2} \sin ^{2} \varphi\right] / \mathrm{W}^{2}\right)
$$

where $2 \mathrm{~W}$ is the $1 / \mathrm{e}$ full amplitude width of the Gaussian shaped beam. Clearly, spillover loss will be important for values of $h$ approaching the radius of the subreflector a.

The central region of the incident wave from the point source is first blocked by the subreflector, and then further truncated by the central blocked area of the primary as it is reflected (see fig.7). Since the 


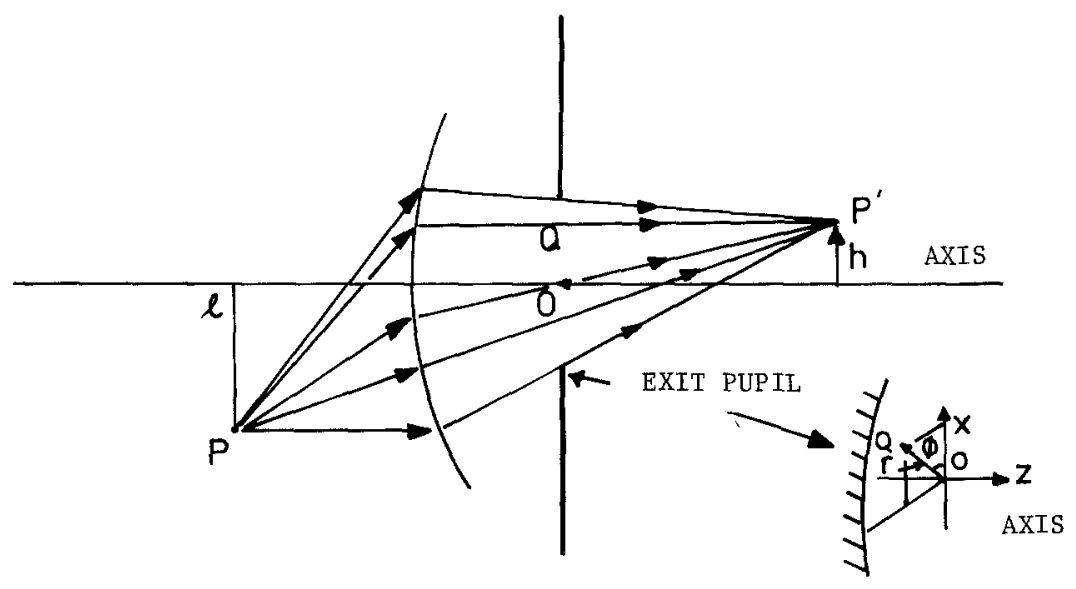

Fig, 5 Simple IMAGing FORMING SYSTEM

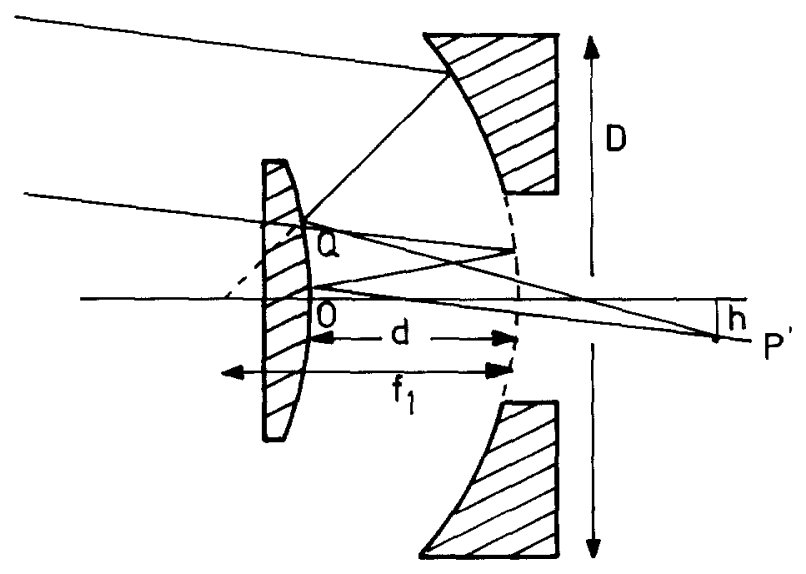

Fig, 6 Cassegrain system 
plane wave is incident at an angle $\theta$, the two truncated central regions are not quite coincident; their centres are separated by $\theta d$, where $d$ is the distance between the mirrors. The image of this doubly truncated form then ends up displaced on the subreflector by a distance of $\epsilon=$ $\theta \mathrm{d}$ and also, of course, the subreflector further truncates the wave at its outer edge. If the subreflector is the same size as the blockage in the primary, the field on the subreflector is non-zero over the area A', as shown in fig.7. However, for a telescope where $\mathrm{f} / \mathrm{f} \mathrm{p}$ is much greater than 1 then $\epsilon=\theta \mathrm{d}=(\mathrm{h} / \mathrm{f}) \mathrm{d} \approx(\mathrm{h} / \mathrm{f}) \mathrm{f}_{\mathrm{p}} \ll \mathrm{h}$ so that the effect of the spillover of the illuminating feed beam at the subreflector dominates over the truncation effects on the incident signal from the point source.

The feeds of the array could be configured so that the feed (h off-axis) is pointed at the centre of the subreflector mirror; in this way truncation effects on the feed beam are reduced at the subreflector, and the beam is more symmetrically positioned with respect to the incident beam (a field lens located immediately in front of the array accomplishes much the same thing - each element of the array sees an optical path consisting of a weak lens and a prism directing its beam towards the centre of the subreflector). We concentrate on the case of a flat array, however, as this type is easier to construct in practice.

In Appendix 5 we derive a relationship for the efficiency with which a feed a distance $h$ from the axis couples to the incoming wave - viz:

$\mathrm{K}(\mathrm{h}) \propto 1-1.95[\mathrm{~F} \lambda / 2 \mathrm{a}]^{2} \cdot \mathrm{q}^{2}-2.6 \times 10^{-3} \cdot[\gamma \lambda / \mathrm{F}(2 \mathrm{a})]^{2} \cdot \mathrm{q}^{4}$

where $q=h / w_{0}$. The term that depends on $q^{2}$ is due to the offset, $h$, of the beam from the feed at the subreflector: the term in $\mathrm{q}^{4}$ is due to the curvature of field.

For a focal plane array where one is prepared to allow no more than a given deterioration in the aperture efficiency this implies a constraint on $\mathrm{h}$ and, therefore, also a constraint on the total number of array elements possible. For a closely packed array the elements are typically separated by a distance equal to the linear size of the aperture required to produce the optimum waist. For a long pyramidal hom, as discussed in [16], the length and height of the hom aperture are approximately $3 W_{0}$ and $2 \mathrm{~W}_{0}$, respectively; while for a long scalar hom the diameter is approximately $3 \mathrm{~W}_{0}$. For the James Clerk Maxwell Telescope (JCMT) [17], for example, $2 \mathrm{a}=750 \mathrm{~mm}, \mathrm{~F}=12, \gamma=33.3$ and at $\lambda=1 \mathrm{~mm}$ the reduction in efficiency is given by:

$\mathrm{K}(\mathrm{h}) \propto 1-(\mathrm{q} / 44.6)^{2}-(\mathrm{q} / 72.6)^{4} \approx 1-(\mathrm{q} / 44.6)^{2}$. 


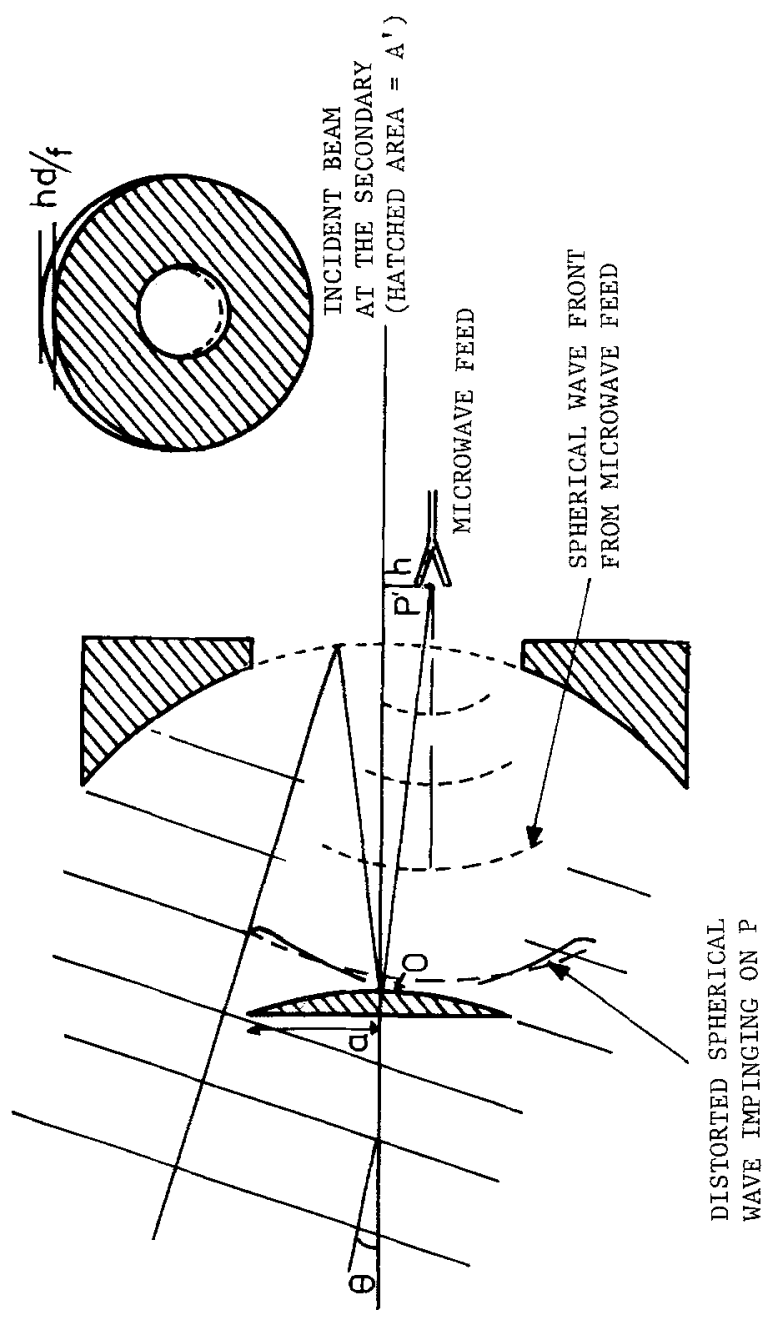


Clearly, spill-over at the subreflector is the more serious constraining factor and for a $10 \%$ reduction in efficiency arrays with radii up to $14 \mathrm{~W}_{\mathrm{O}}$ or a total of about 70 elements are possible (assuming a circular array of scalar horns on a square grid so that the area per element is $9 \mathrm{~W}_{\mathrm{o}}$ ).

For arrays with larger numbers of elements the beams should point at the centre of the subreflector and the array size then becomes limited by the field curvature. The required pointing can be achieved with a field lens of focal length $d_{S}$ in front of the lens ( $d_{S}$ is the distance to the subreflector). In this case for the JCMT, arrays with radii of $41 \mathrm{~W}_{0}$ (or about 580 scalar horn elements maximum) are feasible. Further the curvature of field effects can be reduced by, for example, stepping the thickness of the field lens (as shown in fig.8), so that the optical path difference between the on-axis beam and an off-axis beam is less than the depth of focus. Thus, the first step would occur at a radius of $[\gamma \lambda / \mathrm{F}(2 \mathrm{a}) 19.5]^{-\frac{1}{2}} \cdot 10^{-\frac{1}{4}} \cdot \mathrm{W}_{0}$.

At submillimetre wavelengths for arrays of hom antennas one is usually constrained to use lower F-ratio beams as otherwise the horns become too long and the losses and expense correspondingly high. This implies the use of tertiary optics to refocus the beams to have narrow waists at the array (since $W_{0} \propto F \lambda$ ). Optics for a large array, consisting of a Gaussian telescope[18], are likely to be well approximated by a geometrical system, since the lenses will tend to be positioned in the far field of the array. Thus, the system should be designed so that geometrical coma and astigmatism are minimised, or they will cause deterioration in the quality of the field of view. The array waists will then lie on a Petzval surface whose radius of curvature $R_{p}$ is given by $1 / \mathrm{R}_{\mathrm{p}}=1 / \mathrm{nf}_{1}+1 / \mathrm{nf}_{2}$, where $\mathrm{n}$ is the refractive index of the lens material[15]. This can be chosen to match the field curvature due to the Cassegrain configuration. A field lens will be unnecessary if the position of the first lens of the Gaussian telescope is moved by $\mathrm{f}^{2} / \mathrm{d}_{\mathrm{S}}$; then the central ray of an off axis hom beam is incident on the centre of the subreflector as illustrated in fig.9

\section{IV-Summary and Conclusions.}

1. We have considered in detail mapping of a field with focal plane and aperture plane arrays. As an example, to compare quantitatively the number of hom elements and length of time to observe a field, we assumed a square source of angular extent $\theta_{\mathrm{S}} \times \theta_{\mathrm{S}}$ being mapped by a telescope with a square aperture $D \times D$ fed by an array of long rectangular horns the apertures of which have dimensions $a \times b$. 


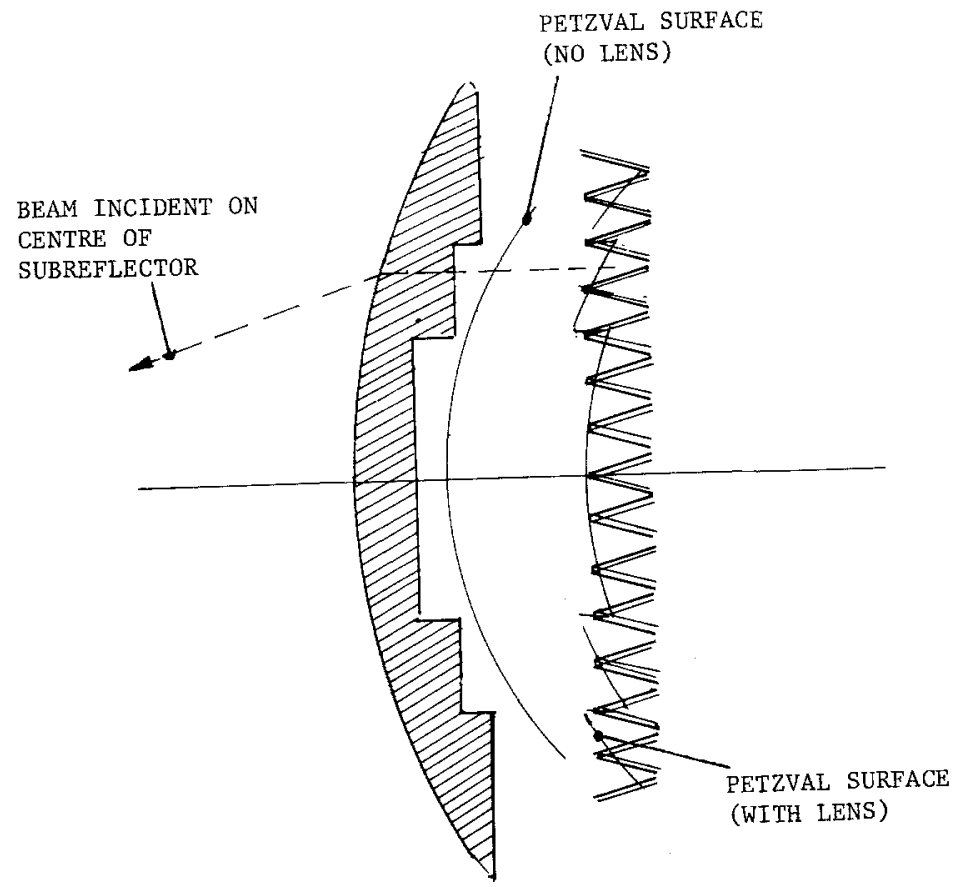

Fig. 8 Field Lens for flat array 


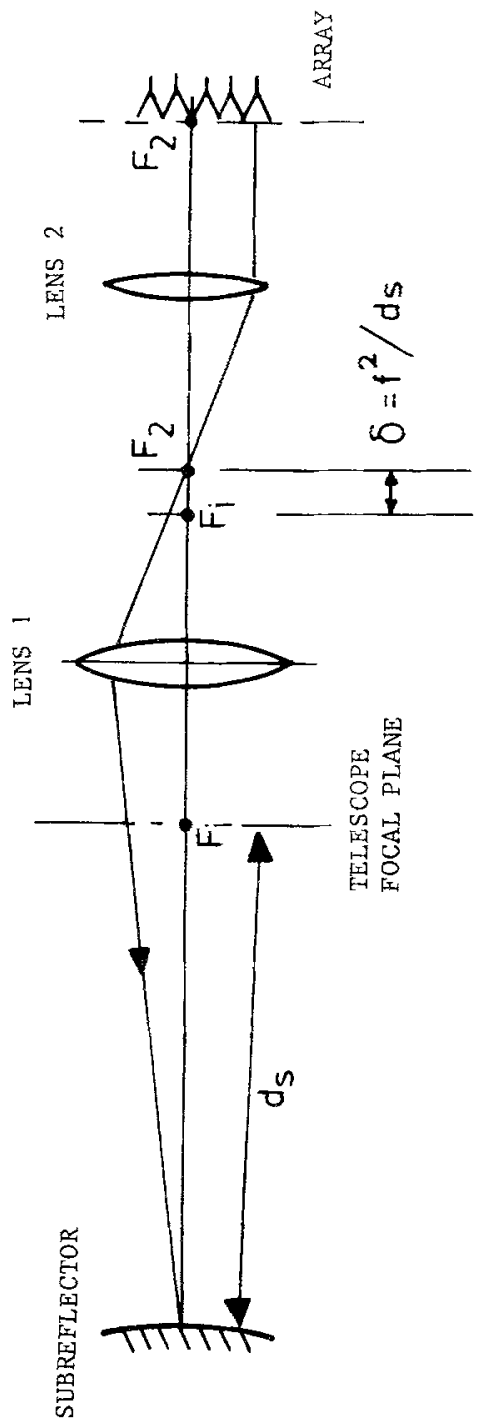

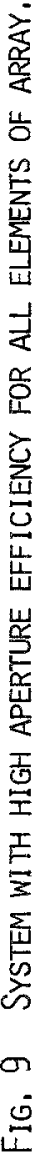


2. In the case of the focal plane array the condition for maximum aperture efficiency is $a=1.88 \mathrm{~F} \lambda$ and $b=1.36 \mathrm{~F} \lambda$, which corresponds to an undersampling factor $U_{f}$ of 10.2. For this array the total number of horns needed to map the source is: $\left[4 . \theta_{\mathrm{s}} /(\lambda / \mathrm{D})\right] / \mathrm{U}_{\mathrm{f}}$.

3. In the case of the aperture plane array of closely packed elements the maximum main lobe sensitivity is given by $8 / \pi^{2}$. The minimum number of homs required to map the source without confusion is $\left[\theta_{\mathrm{S}} /(\lambda / \mathrm{D})\right]^{2}$.

4. With a focal plane array, a source can be mapped to a given signal-to-noise faster if we relax the requirement of maximum aperture efficiency for each individual element of the array and reduce the size of the homs, increasing the number of horns commensurately. Horns with dimensions of $\mathrm{a}=1.31 \mathrm{~F} \lambda$ and $\mathrm{b}=0.96 \mathrm{~F} \lambda$ give a reduction in aperture efficiency of $18 \%$ but a reduction in the undersampling factor by 2 , thus decreasing the overall time to map a source by $28 \%$.

5. The time taken to map a source using an aperture plane array can be similarly reduced by using only the central beams and using a hybrid mapping technique whereby the telescope is moved to cover the entire source. However, this implies either increasing the total number of array elements (to give a bigger field, and hence lower losses anyway) or accepting the resultant confusion and applying a deconvolution technique to the image.

6. We conclude that, with the possible exception of the number of elements required to map a field, the advantages of the focal plane array over an aperture plane array far outweigh the disadvantages for most purposes. The focal plane array has simpler electronics, fewer restrictions on the field of view, and lends itself to an easier form of "hybrid" mapping (when the telescope is moved to compensate for undersampling of either image or beam space).

7. The field of view of a Cassegrain telescope was considered for the case of a focal plane array of feeds which have Gaussian shaped beams. For a flat array off-axis aberrations (coma, astigmatism and curvature of field) and spill-over at the subreflector contribute to the deterioration in aperture efficiency, and their effect on the maximum size of an array was quantified.

\section{Acknowledgements}

The authors would like to express their gratitude to Dr. P.J.Duffett-Smith and Dr. P.F.Scott for their helpful comments in preparing this manuscript. 


\section{APPENDIX 1 Glossary of terms}

Section II and Appendix 2:

$\underline{\text { Plane }}$

sky

aperture

focal plane

image plane coordinates

$\theta$
Quantities

$e_{s}$ (field of source)

$B$ (brightness $\left.=\left|e_{s}\right|^{2}\right)$

$\mathbf{u}(=\mathbf{x} / \lambda)$

$r\left(=x / f_{t}\right)$

$\rho\left(=f_{1} x / f_{t} \lambda\right)$

E (source fields)

b synthesis array baseline

$B$ source brightness

$\hat{B} \operatorname{map}$

d interelement separation

D telescope diameter

$f_{1}$ focal length of tertiary lens

$f_{t}$ telescope effective focal length

$F$ telescope focal ratio

$G$ telescope power pattern

h fields at horn phase centre

H horn far-field pattern

$\mathrm{m}, \mathrm{n}$ indices

$\mathrm{P}_{\mathrm{i}} \quad\left|\mathrm{v}_{\mathrm{i}}\right|^{2}$

$s, t$ inter-element spacings in phased array

$U$ far field of array of isotropic radiators

$v$ (i) voltage from horn or array

$V$ visibility

$\gamma \quad\left(=-\mathrm{f}_{\mathrm{t}} / \mathrm{f}_{1}\right)$

$\lambda$ wavelength of radiation

II $\quad\left(=U^{*} w\right)$

$\varphi_{m n}$ phase increment for mnth element of array 
Section III and Appendix 3 :

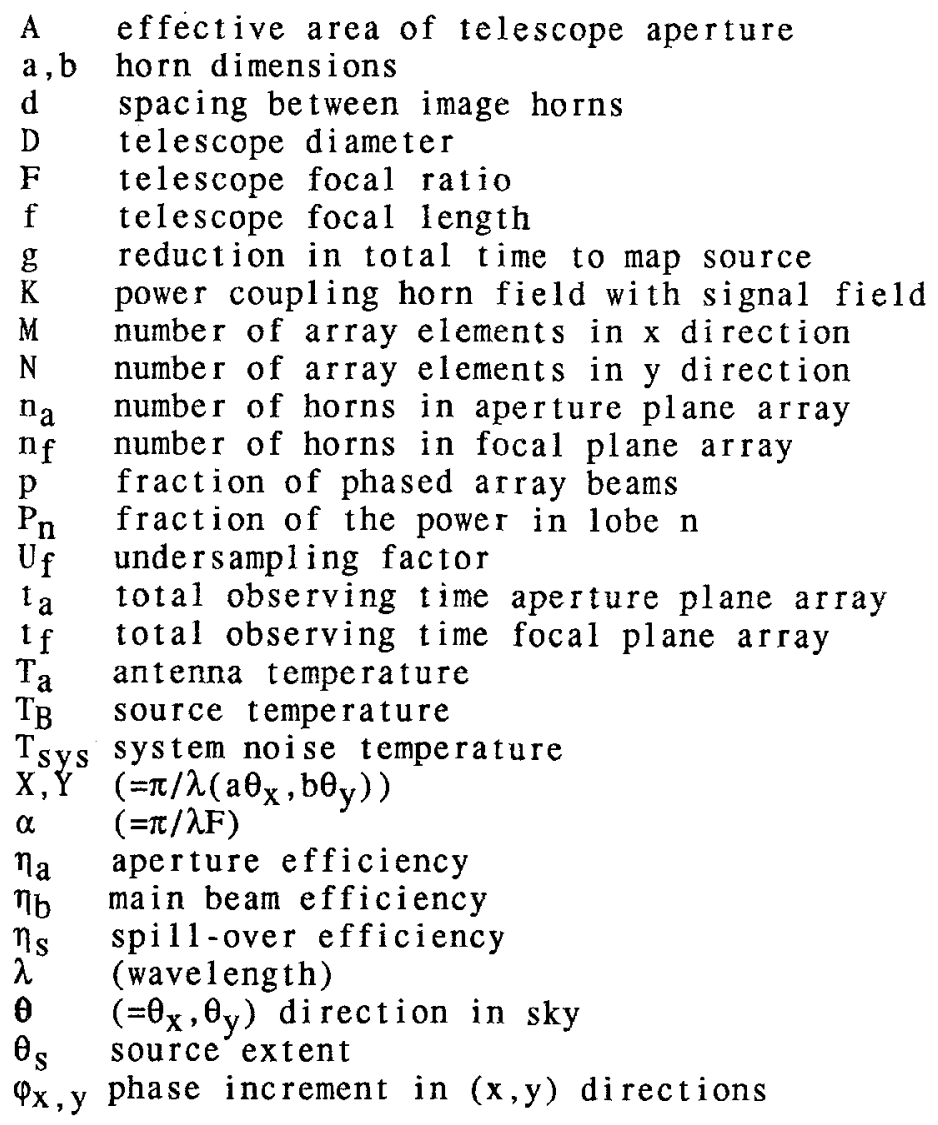




\section{Section IV and Appendices 4 and 5:}

A area of the subreflector

a radius of subreflector

$b$ radius of blockage at secondary

C astigmatism coefficient

D diameter of primary

$D$ curvature of field coefficient

$\mathrm{d}_{\mathrm{S}}$ subreflector horn distance

E distortion coefficient

$E_{S}$ source field at subreflector

$\mathrm{E}_{\mathrm{g}}$ field due to horn at subreflector

$F$ coma coefficient

F telescope focal ratio

$f$ effective focal length of telescope

$f_{p}$ focal length of primary

$\mathrm{g} \quad(=\rho \cdot \exp \rho /[1-\exp \rho])$

$h$ distance of array element off-axis

$I_{0}$ modified Bessel function of order zero

$\mathrm{k} \quad(=2 \pi / \lambda)$

$\mathrm{K}$ coupling efficiency

$P$ point off-axis

q $\quad\left(=H / w_{O}\right)$

Q point on exit pupil

$\hat{\mathrm{r}} \quad(=(\mathrm{D} / 2 \mathrm{a}) \cdot \mathrm{r})$

$R_{p}$ Petzval surface radius of curvature

W $1 / \mathrm{e}$ in amplitude beam radius

$W_{0} \quad W$ at the horn phase centre

$\alpha \quad(=h / f)$

$\beta \quad\left(=\gamma\left(h k / 32 F^{3}\right)(h / a)(W / a)^{2}\right.$

$\gamma \quad\left(=\mathrm{f} / \mathrm{f}_{\mathrm{p}}\right)$

$\epsilon$ off-axis beam displacement on subreflector

p $\quad\left(=(r / W)^{2}\right)$

$\theta \quad$ angle of source of $f$-axis

$\Phi$ aberration function 
APPENDIX 2 Focal plane and aperture plane arrays

\section{Focal plane arrays.}

Fourier optics can be used to examine the effect of diffraction and vignetting on the radiation from the sky at the focal plane. Suppose the fields - due to radio sources in the sky - at the aperture plane of a telescope are given by $E_{a}(u)$, where $u$ is some point on the plane defined by the telescope aperture and the origin is taken to lie on the telescope axis (see fig.2). We can write the aperture fields in terms of a sum of incident plane waves with appropriate phase terms, the relative amplitude of the wave in the direction $\theta$ being $\mathrm{e}_{\mathrm{S}}(\boldsymbol{\theta})$. Thus we get:

$$
E_{\mathrm{a}}(\mathbf{u})=\int \mathrm{dA}_{\boldsymbol{\theta}} \mathrm{e}_{\mathrm{S}}(\boldsymbol{\theta}) \exp \{-2 \pi \mathrm{j} \mathbf{u} \cdot \boldsymbol{\theta}\}
$$

To simplify the algebra $\mathbf{u}$ been made dimensionless by expressing the distance in wavelengths. The sky brightness (the intensity of the radiation from the $\theta$ direction) is given by $B(\theta) \alpha\left|e_{S}(\theta)\right|^{2}$.

Assuming that the effective focal length of the telescope is much greater than its aperture diameter, the signal fields at the aperture and focal planes of the telescope are a Fourier pair, so that we have:

focal plane $\mathrm{e}(\mathbf{r})=\int \mathrm{dA}_{\mathrm{u}} \mathrm{E}_{\mathrm{a}}(\mathbf{u}) \mathrm{W}(\mathbf{u}) \exp \{-2 \pi \mathrm{j} \mathbf{r} \cdot \mathbf{u}\}$

aperture plane $\mathrm{E}_{\mathrm{a}}(\mathbf{u}) \mathrm{W}(\mathbf{u})=\int \mathrm{dA}_{\mathrm{r}} \mathrm{e}(\mathbf{r}) \exp \{2 \pi \mathrm{j} \mathbf{r} \cdot \mathbf{u}\}$

W(u), the telescope aperture weighting function, represents the truncation of the incident beam at the telescope aperture, and $w(r)$ is its Fourier transform. In fact, $w(\mathbf{r})$ is the intrinsic field pattern of the telescope, or the far field pattern produced by the uniformly illuminated aperture. e(r) is the field due to the source at the telescope focal plane.

Note that $\mathbf{r}$ is the distance from the axis divided by $f_{t}$ (thus, $r$ is dimensionless) and we define the forward Fourier transform (along the propagation axis $\mathrm{z}$ ) such that the argument of the exponential is negative; the reverse transform therefore has a positive sign.

We consider an array of horns placed on a part of telescope focal plane where there is an undistorted diffraction limited image of the sky. For the $i$ th horn we write the virtual fields (those which would exist if the fields in the aperture of the hom were propagated back to its phase centre in free space) as $h_{\mathbf{i}}(\mathbf{r})$. If all the homs are identical then: $h_{\mathbf{i}}(\mathbf{r})=$ $h\left(\mathbf{r}-\mathbf{r}_{\mathbf{i}}\right)$. Thus, the voltage output available at the terminals of hom "i" will be: 


$$
\mathrm{v}_{\mathrm{i}}=\int \mathrm{dA}_{\mathrm{r}} \mathrm{h}_{\mathrm{i}}^{*}(\mathbf{r}) \mathrm{e}(\mathbf{r})
$$

and so the power coupled to horn " $\mathrm{i}$ " is:

$$
P_{i}=\left\langle\left|v_{i}\right|^{2}\right\rangle
$$

where angle brackets denote time average. This is equivalent to the response of the antenna to a celestial source and is given by the convolution of the antenna power pattern with the source intensity distribution, i.e.:

$$
P_{i}=\int B(\theta) G\left(\theta_{i}-\mathbf{r}\right) d A
$$

where $\mathrm{G}(\mathbf{r})=|F T\{\mathrm{H}(-\mathbf{u}) \cdot \mathrm{W}(-\mathbf{u})\}|^{2}$ and $\mathrm{H}(\mathbf{u})$ is the far field pattern of a hom with "phase centre" fields $h(\mathbf{r}) . \mathrm{G}(\mathbf{r})$ has the same functional form as the intensity or power pattern of the horn on the sky (i.e is the squared modulus of the far fields of the truncated hom beam at the telescope aperture). A particular point $r$ in the focal plane is related to a particular direction $\boldsymbol{\theta}=-\mathrm{r}$ on the sky.

\section{Aperture plane arrays}

\section{(i) Phased array}

Consider a regular array of $M \times N$ horn-fed mixer elements in the aperture plane of the telescope; the (dimensionless) distance between the elements in the $u_{x}$ direction is $s=d_{x} \lambda$ and in the $u_{y}$ direction is $t=$ $\mathrm{d}_{\mathrm{y}} \wedge$. The outputs of the array are added together, as shown in fig. 3 , with a linear phase delay $\theta_{1}\left(=\left(\theta_{i x}, \theta_{i y}\right)\right)$ applied in the $u_{x}$ and $u_{y}$ directions so that for the $(\mathrm{m}, \mathrm{n})$ th element at position $\mathrm{u}_{\mathrm{mn}}=(\mathrm{ms}, \mathrm{nt})$ the phase with respect to a fictitious $(0,0)$ point can be represented as:

$$
\varphi_{\mathrm{mn}}\left(\boldsymbol{\theta}_{\mathrm{i}}\right)=2 \pi\left(\mathbf{u}_{\mathrm{mn}} \cdot \boldsymbol{\theta}_{\mathrm{i}}\right)
$$

Then the output voltage from the array due to the signal fields $\mathrm{E}_{\mathrm{a}}$ at the telescope aperture is given by:

$\mathbf{v}\left(\boldsymbol{\theta}_{\mathrm{i}}\right)=\sum_{m=1}^{M} \sum_{n=1}^{N}\left(\int d A_{\mathbf{u}} \cdot h_{m n}(\mathbf{u}) \cdot W(\mathbf{u}) \cdot E_{a}^{*}(\mathbf{u})\right) \exp \left(-2 \pi j \mathbf{u}_{m n} \cdot \boldsymbol{\theta}_{\mathbf{i}}\right)$

where $\left(\int_{d A_{u}} h_{m n}(u) . W(u) \cdot E_{a}^{*}(u)\right)$ represents the coupling of the fields of the image detector antenna $h_{m n}(u)$ to the signal field $E_{a}(u)$ in the plane of the aperture. W(u) is the telescope aperture weighting function as before. If the image array of horns is of physical extent less than the telescope aperture then $\mathrm{W}(\mathrm{u})$ is uniform over the array and can be ignored. Outside this region, $W(u)$ is zero and so represents the 
truncation of the image array by the finite extent of the telescope aperture.

For an array of identical elements, where the virtual fields at the element phase centres are $h_{m n}(u)=h\left(u-u_{m n}\right),(2.6)$ can be written:

$$
\begin{aligned}
& v\left(\boldsymbol{\theta}_{\mathrm{i}}\right)=\sum_{m=1}^{M} \sum_{n=1}^{N} \int d A_{u} h\left(\mathbf{u}-\mathbf{u}_{m n}\right) \cdot E_{a}^{*}(\mathbf{u}) \cdot W\left(\mathbf{u}_{m n}\right) \cdot \exp \left(-2 \pi j \mathbf{u}_{m n} \cdot \boldsymbol{\theta}_{i}\right) \\
& =\int d_{A} A_{u} \cdot \int d_{A} A^{\prime} \cdot h\left(\mathbf{u}-\mathbf{u}^{\prime}\right)\left[\Sigma_{m, n} \delta\left(\mathbf{u}^{\prime}-\mathbf{u}_{m n}\right) \cdot W\left(\mathbf{u}_{m n}\right)\right] \\
& \times E_{a}^{*}(\mathbf{u}) \cdot \exp \left(-2 \pi j \mathbf{u}^{\prime}, \boldsymbol{\theta}_{\mathrm{i}}\right) \\
& =\int \mathrm{dA}_{\mathbf{u}^{\prime}} \cdot\left[\sum_{\mathrm{m}, \mathrm{n}} \delta\left(\mathbf{u}^{\prime}-\mathbf{u}_{\mathrm{mn}}\right)\right] \cdot \exp \left(-2 \pi \mathrm{j} \mathbf{u}^{\prime} \cdot \boldsymbol{\theta}_{\mathrm{i}}\right) \\
& \times \int \mathrm{dA}_{\mathrm{u}}, \mathrm{h}\left(\mathbf{u}-\mathbf{u}^{\prime}\right) \cdot \mathrm{E}_{\mathrm{a}}^{*}(\mathbf{u})
\end{aligned}
$$

By the convolution theorem we can write $v\left(\theta_{1}\right)$ as the convolution of

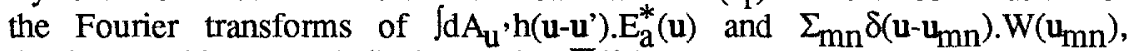
the latter which we shall denote by $\Pi\left(\theta_{i}\right)$.

The Fourier transform of $\int \mathrm{dA}_{\mathbf{u}}, \mathbf{h}\left(\mathbf{u}-\mathbf{u}^{\prime}\right) \cdot \mathrm{E}^{*}(\mathbf{u})$ is the product of the far field pattern of the image hom $H(\theta)$ and the incident signal field from the direction $\theta, \mathrm{e}_{\mathrm{S}}(\boldsymbol{\theta})$, yielding

$$
\mathrm{v}\left(\boldsymbol{\theta}_{\mathrm{i}}\right)=\int \mathrm{d} A_{\theta} \Pi\left(\boldsymbol{\theta}^{\prime}-\boldsymbol{\theta}_{\mathrm{i}}\right) \cdot \mathrm{H}(\boldsymbol{\theta}) \cdot \mathrm{e}_{\mathrm{S}}(\theta)
$$

Squaring and taking the time average using a procedure similar to that applied to the focal plane array, we find that the total power $P\left(\theta_{1}\right)$ is given by:

$$
\mathrm{P}\left(\boldsymbol{\theta}_{\mathrm{i}}\right)=\int \mathrm{dA}_{\theta} \mathrm{B}(\boldsymbol{\theta})|\mathrm{H}(\boldsymbol{\theta})|^{2} \cdot\left|\mathrm{G}\left(\boldsymbol{\theta}-\boldsymbol{\theta}_{\mathrm{i}}\right)\right|^{2}
$$

where $B(\theta)=\left|e_{S}(\theta)\right|^{2}$ and $G(\theta)=|\Pi(\theta)|^{2}$ is the source field.

$G$ is the fourier transform of $\Sigma_{m n} \delta\left(\mathbf{u}-u_{m n}\right) . W\left(u_{m n}\right)$ and so can be written as a convolution of $\mathrm{U}(\boldsymbol{\theta})$, the Fourier transform of a 2-dimensional array of $\delta$-functions and $w(\theta)$, the Fourier transform of $W(\theta)$ - the truncation function for the array. $U(\theta)$ has the form of the far field of a phased array of isotropic radiators. 
(ii) Synthesis array.

The telescope is pointed at some point $\theta_{0}$ in the source field. We define the baseline $\mathbf{b}_{\mathbf{i}}=\Delta \mathbf{u}_{\mathbf{i}}=\mathbf{u}_{i_{1}}-\mathbf{u}_{i_{2}}$, to be the spacing (in the aperture plane) of the images of the two elements of the interferometer (which is physically located at the image of the aperture plane as in fig.1). Then, if $\boldsymbol{\theta}$ is the position of the source and assuming $\boldsymbol{\theta}_{\mathrm{o}} \cdot \mathbf{b}_{1}=0$, the output from the correlator is given by:

$\mathrm{V}\left(\mathbf{b}_{\mathrm{i}}\right)=\int \mathrm{dA}_{\boldsymbol{\theta}} \mathrm{B}(\boldsymbol{\theta})|\mathrm{H}(\boldsymbol{\theta})|^{2} \cdot \mathrm{W}_{\mathrm{b}}\left(\mathbf{b}_{\mathrm{i}}\right) \cdot \exp \left(2 \pi j \mathbf{b}_{\mathrm{i}} \cdot \boldsymbol{\theta}\right)$

where $H(\theta)$ is the far field pattern of the image of the horn as before. The factor $W_{b}\left(b_{i}\right)=W_{b}\left[b\left(u_{i_{2}}, u_{i_{1}}\right)\right]=W\left(u_{i_{2}}\right) \cdot W\left(u_{i_{1}}\right)$ ensures that the interferometer pair does not contribute to the final signal if either image element lies outside the physical aperture of the telescope.

Noting that $V(-b)=V^{*}(b)$, we can then write the visibility-plane distribution in terms of the samples $V_{i}$ as:

$\mathrm{V}(\mathbf{b})=\Sigma_{\mathrm{i}}\left[\mathrm{V}_{\mathrm{i}} \delta\left(\mathbf{b}-\mathbf{b}_{\mathrm{i}}\right)+\mathrm{V}_{\mathrm{i}}^{*} \delta\left(\mathbf{b}+\mathbf{b}_{\mathrm{i}}\right)\right]$

The original brightness distribution, $\mathrm{B}(\boldsymbol{\theta})$, is often estimated from $\mathrm{V}(\mathrm{b})$ by taking the inverse Fourier Transform. Then (ignoring subtleties such as gridding) we have:

$$
\hat{B}(\boldsymbol{\theta})=\int \mathrm{dA}_{\mathbf{b}} \cdot \mathrm{V}(\mathbf{b}) \cdot \exp \{-2 \pi \mathrm{j} \mathbf{b} \cdot \boldsymbol{\theta}\}
$$

Substituting $V(b)$ and $V_{i}$ using (2.10) and (2.11) we find that:

$$
\begin{aligned}
& \hat{\mathrm{B}}(\boldsymbol{\theta})=\int \mathrm{dA}_{\theta}, \mathrm{B}\left(\boldsymbol{\theta}^{\prime}\right)\left|\mathrm{H}\left(\theta^{\prime}\right)\right|{ }^{2} \sum_{\mathrm{i}} \mathrm{W}_{\mathrm{b}}\left(\mathrm{b}_{\mathrm{i}}\right) \cdot \cos \left[2 \pi \mathrm{b}_{\mathrm{j}} \cdot\left(\boldsymbol{\theta} \cdot \boldsymbol{\theta}^{\prime}\right)\right] \\
& \hat{O} \mathrm{r} \\
& \hat{\mathrm{B}}(\boldsymbol{\theta})=\left\{\mathrm{B}(\boldsymbol{\theta})|\mathrm{H}(\boldsymbol{\theta})|^{2}\right\} *\left\{\sum_{\mathrm{j}} \mathrm{W}_{\mathrm{b}}\left(\mathrm{b}_{\mathrm{i}}\right) \cdot \cos \left[2 \pi \mathrm{b}_{\mathbf{i}} \cdot \theta\right]\right\} .
\end{aligned}
$$

That is, the true source distribution $B(\theta)$ has been multiplied by the primary beam power pattern $\left|\mathrm{H}_{\mathrm{i}}(\boldsymbol{\theta})\right|^{2}$ and convolved with the synthesised beam. We can rewrite this in terms of the array-plane quantity $\rho$ and the magnification factor $\gamma=-f_{1} / f_{t}$ as:

$\hat{\mathbf{B}}(\boldsymbol{\theta})=\left\{B(\theta)|\mathrm{H}(\theta)|^{2}\right\} *\left\{\sum_{\mathrm{i}} W\left(\gamma \rho_{\mathrm{i}_{1}}\right) \cdot W\left(\gamma \rho_{\mathrm{i}_{2}}\right) \cdot \cos \left[2 \pi\left(\rho_{\mathrm{i}_{1}}-\rho_{\mathrm{i}_{2}}\right) \cdot \theta\right]\right\}$ 
APPENDIX 3 Derivations of foward coupling and time to map a source

\subsection{Focal plane array}

\section{Coupling Efficiency}

In a focal plane array one normally optimises the aperture efficiency for each horn of the array (the coupling between the horn and a point source situated along the optic axis for that horn). In this case it is easiest to perform the coupling integral (between the fields radiated by the horn and the incoming field from a point source) at the hom mouth. A point source at infinity produces a top-hat distribution at the square aperture of the antenna which produces a sinc-like distribution in both the $\mathrm{E}$ and $\mathrm{H}$ directions in the focal plane. The coupling $\mathrm{K}$ is given by:

$K=\frac{\left[\iint_{H^{d}} d A^{\prime} \cos \left(\pi x^{\prime} / a\right) \operatorname{sinc}\left(\alpha x^{\prime}\right) \operatorname{sinc}\left(\alpha y^{\prime}\right)\right]^{2}}{\left[\iint_{H} d A^{\prime} \cos ^{2}\left(\pi x^{\prime} / a\right)\right]\left[\iint_{-\infty}^{+\infty} d A^{\prime} \operatorname{sinc}^{2}\left(\alpha x^{\prime}\right) \operatorname{sinc}^{2}\left(\alpha y^{\prime}\right)\right]}$

where $\alpha=\pi / \lambda \mathrm{F}, \mathrm{dA}^{\prime}=\mathrm{dx} \mathrm{x}^{\prime} \mathrm{y}^{\prime}$ and $\iint_{\mathrm{H}} \equiv \int_{-a / 2}+\mathrm{a}_{2}[\mathrm{~b} / 2 / 2$. This can be rewritten as:

$\mathrm{K}=\frac{2 \alpha^{2} a b}{\pi^{6}}\left|\int_{0}^{\frac{1}{2} \pi} \mathrm{d} \psi \operatorname{sinc}(\alpha b \psi / \pi)\right|^{2} \cdot\left|\int_{0}^{\frac{1}{2} \pi} \mathrm{d} \psi \cdot \cos \psi \cdot \operatorname{sinc}(\alpha a \psi / \pi)\right|^{2}$

$\mathrm{K}$ has a maximum when $\alpha \mathrm{a} / \pi=1.88$ and $\alpha \mathrm{b} / \pi=1.36$, giving a ratio for $a / b$ of 1.38 with a coupling $K$ given as 0.745 . Since $\alpha$ depends on the focal ratio only, $a$ and $b$ are thus fixed by $F$ :

$$
\begin{aligned}
& \alpha \mathrm{a} / \pi=1.88 \text { gives } \mathrm{a}=1.88 \pi / \alpha=1.88 \lambda \mathrm{F} \\
& \alpha \mathrm{b} / \pi=1.36 \text { gives } \mathrm{b}=1.36 \pi / \alpha=1.36 \lambda \mathrm{F}
\end{aligned}
$$

The Nyquist sampling rate is $\frac{1}{2} \mathrm{~F} \lambda$, so that for a physically realizable array the undersampling factor $\mathrm{U}_{\mathrm{f}}$ is given by:

$$
\mathrm{U}_{\mathrm{f}} \geqslant 4 \mathrm{ab} /(\mathrm{F} \lambda)^{2}
$$

In this case the undersampling factors are 2.72 along the $y$-axis (E-plane) and 3.76 along the $\mathrm{x}$-axis (H-plane) giving an overall undersampling factor of $\mathbf{1 0 . 2}$ 


\section{Spillover}

At the telescope the beams are truncated at the edge of the square aperture. Along the E-plane the distribution is $\sin Y / Y$ where $Y=$ $\pi$ by $/ \lambda \mathrm{f}$. The fraction of the power lost to spill-over is then:

$$
1-\int_{0}^{0} .68 \pi(\sin Y / Y)^{2} d Y / \int_{0}^{\infty}(\sin Y / Y)^{2} d Y
$$

Similarly along the H-plane the distribution is $\cos \mathrm{X} /\left[1-\left(\mathrm{X} / \frac{1}{2} \pi\right)^{2}\right]$ where $\mathrm{X}=\pi \mathrm{ay} / \lambda \mathrm{f}$. The spillover efficiency is therefore given by:

$$
\eta_{\mathrm{s}}=\frac{8}{\pi^{3}} \cdot \int_{0}^{X_{m_{d}}} \mathrm{dX} \int_{0}^{Y_{m_{d Y}}}\left|\frac{\cos ^{2} X}{\left[\left(\frac{1}{2} \pi\right)^{2}-X^{2}\right]^{2}} \cdot \frac{\sin ^{2} Y}{Y^{2}}\right|
$$

where $\left(X_{m}, Y_{m}\right)=\pi / 4 F \lambda .(a, b) . X$ is truncated at $X=0.94 \pi$ and $Y$ at $\mathrm{Y}=0.68 \pi$ corresponding to an edge taper of $-8.1 \mathrm{~dB}$ in both planes. The total spill-over efficiency therefore is $\eta_{\mathrm{S}}=0.831$.

The extent of the image of the source in the telescope focal plane is given by $f \theta_{S} \times f \theta_{S}$. Therefore, the number of homs $n_{f}$ in the focal plane array is given by:

$$
\begin{aligned}
\mathrm{n}_{\mathrm{f}} & =\mathrm{f}^{2} \theta \mathrm{g} / \mathrm{ab} \\
& =\mathrm{f}^{2} \theta_{\mathrm{S}}^{2} / 1.36 \times 1.88 \cdot\left(\mathrm{F}^{2} \lambda^{2}\right) \\
& =(1 / 2.55) \cdot \theta_{\mathrm{S}} /(\lambda / \mathrm{D})^{2}
\end{aligned}
$$

If the focal plane were fully sampled, ignoring the penalty in aperture efficiency, than the total number of homs required would be $4 . \theta_{\mathrm{S}}^{2} /(\lambda / \mathrm{D})$.

Time to map a source

For $U_{f}=4 a b /(F \lambda)^{2}$, the time to map a source $t$ is given by:

$t \propto U_{f} / \eta_{\mathrm{S}}=\pi^{2} X_{m} Y_{m} \cdot\left[\int_{0}^{X_{m_{d X}}} \int_{0}^{Y_{m_{d}}}\left|\frac{\cos ^{2} X}{\left[\left(\frac{1}{2} \pi\right)^{2}-X^{2}\right]^{2}} \cdot \frac{\sin ^{2} Y}{Y^{2}}\right|_{3 \cdot 7)}^{-2}\right.$

where $\left(\mathrm{X}_{\mathrm{m}}, \mathrm{Y}_{\mathrm{m}}\right)=\pi / 4 \mathrm{~F} \lambda .(\mathrm{a}, \mathrm{b})$ as before. The minimum mapping time is achieved for horns having $\mathrm{a}=1.305 \mathrm{~F} \lambda$ and $\mathrm{b}=0.96 \mathrm{~F} \lambda$ (corresponding to an undersampling factor of 5.0) with a decrease in aperture efficiency to 0.820 times the maximum aperture efficiency. This minimum time is 0.720 times that using the maximum aperture efficiency criterion. It might be argued that we should be using $\eta_{a}$ rather than $\eta_{s}$ in the above expression, in order to maximise the transfer functions for source variation on a scale equal to the telescope resolution. The calculation proceeds along similar lines; however, it is 
easy to see that as we reduce the size of the horn $\eta_{\mathbf{a}}$ asymptotically approaches $\eta_{S}$, so we could expect the optimum values of $a$ and $b$ to be very similar.

\subsection{Aperture-plane array.}

\section{Coupling efficiency}

We consider an aperture plane array consisting of an array of $\mathrm{N} \times \mathrm{M}$ elements. The phase increments per half element ("phase slopes") in the $x$ and $y$ directions are $\varphi_{x}=\pi \operatorname{acos} \theta_{i x} / \lambda$ and $\varphi_{y}=\pi b \cos \theta_{i y} / \lambda$, respectively - see equation (2.5). Then the field produced by the array is of the form:

$E(X, Y: \varphi)=C \cdot\left[\frac{\cos X \cdot \sin Y}{\left[1-\left(X / \frac{1}{2} \pi\right)^{2}\right] \cdot Y}\right]\left[\frac{\sin \left(M\left(X-\varphi_{X}\right)\right) \cdot \sin \left(N\left(Y-\varphi_{y}\right)\right)}{\sin \left(X-\varphi_{X}\right) \cdot \sin \left(Y-\varphi_{y}\right)}\right]$

where $(X, Y)=\pi / \lambda \cdot\left(\operatorname{acos} \theta_{X}, b \cos \theta_{y}\right)$. Thus, the direction cosines in the $H$ and $E$ planes in the far field are $\left(\cos \theta_{X}, \cos \theta_{y}\right)$, respectively.

We can separate this expression into E-plane and $\mathrm{H}$-plane components. First we treat the E-plane component:

$E \propto[\sin Y / Y] \cdot\left[\sin \left(M\left(Y-\varphi_{y}\right)\right) / \sin \left(Y-\varphi_{y}\right)\right]$.

Assuming $M$ is large then $\sin Y / Y$ does not vary over an individual lobe of $\left[\sin \left(\mathrm{M}\left(\mathrm{Y}-\varphi_{\mathrm{y}}\right)\right) / \sin \left(\mathrm{Y}-\varphi_{\mathrm{y}}\right)\right]$ and it is possible to calculate the power in the nth grating lobe as approximately the square of the primary beam amplitude at each point where the grating lobe occurs, multiplied by the normalised power of each grating lobe - i.e., after some tedious manipulation:

$P_{n}=\left(\sin \left(n \pi+\varphi_{X}\right) / n \pi+\varphi_{X}\right)^{2} \int_{-\infty}^{\infty}\left(\sin M Y^{\prime} / Y^{\prime}\right)^{2} d Y^{\prime}$

The fraction of the total power in the zero order lobe is then given by: $\left(P_{0} / \Sigma P_{n}\right)_{E}=\operatorname{sinc}^{2} \varphi_{x}$. Similarly along the $H$-plane the fraction of the total power in the zero order mode is given by: $\left(P_{0} / \Sigma P_{n}\right)_{H}=$ $\left(8 / \pi^{2}\right) \cdot \cos ^{2} \varphi_{x} /\left(1-\left(2 \varphi_{x} / \pi\right)^{2}\right)^{2}$. Combining these two results we find that the fraction of the power in the main (zero-order) lobe is given by:

$$
\eta_{b}\left(\varphi_{x}, \varphi_{y}\right)=\frac{8}{\pi^{2}} \quad\left[\frac{\sin \varphi_{y}}{\varphi_{y}}\right]^{2} \cdot\left[\frac{\cos \varphi_{x}}{1-\left(\varphi_{x} / \frac{1}{2} \pi\right)^{2}}\right]^{2}
$$


$\eta_{\mathrm{b}}$ has a maximum value given by $\eta_{\mathrm{bmax}}=8 / \pi^{2}=0.8106$.

In order that there are no aliasing effects when a source is being mapped none of the higher order lobes should intersect the source. For a square source of angular extent $\theta_{\mathrm{S}} \times \theta_{\mathrm{S}}$, this implies a maximum $\varphi_{\mathrm{X}}$ and $\varphi_{\mathrm{y}}$ of $\frac{1}{2} \pi$, (see fig.4). At the corner of the source where $\varphi_{\mathrm{x}}=$ $\varphi_{\mathrm{y}}=\frac{1}{2} \pi, \eta_{\mathrm{b}}$ takes on its minimum value given by $\eta_{\mathrm{bmin}}=0.2026$.

\section{No of horns in the array.}

The required beam spacing is given by $(\lambda / 2 \mathrm{D})^{2}$ and the total number of beams to map the $\theta_{\mathrm{S}} \times \theta_{\mathrm{S}}$ source is $4 . \theta_{\mathrm{S}} /(\lambda / \mathrm{D})^{2}$. The number of horns in an aperture plane array is set by the fact that the maximum allowed phase slopes to avoid aliasing (see previous paragraph) are $\varphi_{\mathrm{X}}$ $=\varphi_{\mathrm{y}}= \pm \frac{1}{2} \pi$ giving the source extent in the $\mathrm{x}$ and $\mathrm{y}$ directions of $\theta_{\mathrm{x}}$ $=\lambda / \mathrm{a}, \theta_{\mathrm{y}}=\lambda / \mathrm{b} ; \theta_{\mathrm{s}}^{2}=\lambda^{2} / \mathrm{ab}=\mathrm{MN} \lambda^{2} / \mathrm{D}^{2}$ (since $\varphi_{\mathrm{x}}=\pi \mathrm{a} \theta_{\mathrm{x}} / \lambda, \varphi_{\mathrm{y}}=$ $\pi \mathrm{b} \theta_{\mathrm{y}} / \lambda$ and $\mathrm{D}=\mathrm{Ma}=\mathrm{Nb}$ ). Thus, the total number of homs in the aperture plane $=\theta_{\mathrm{S}}^{2} /(\lambda / \mathrm{D})^{2}$

\section{Time to map a source}

If use a fraction $p \times p$ of the array of beams, then $\eta_{b m i n}$ (the minimum sensitivity for the subset of beams now being used) is given by $\eta_{b}\left(p_{\frac{1}{2}} \pi, p_{\frac{1}{2}} \pi\right)$ and the undersampling factor is $1 / \mathrm{p}^{2}$. The relative time it would take to map the source would then be given (from equation (22) by: $\mathrm{t} \propto$ undersampling factor $\times 1 / \mathrm{m}_{\mathrm{bmin}}{ }^{2}$ ). The total time to map the source is reduced by a factor $g$, where:

$$
g=\left[\frac{\pi p^{\frac{1}{2}}\left(1-p^{2}\right)}{2 \operatorname{sinp} \pi}\right]^{4}
$$

It turns out that this has a minimum when $\mathrm{p}=0.605$ which gives $\mathrm{g}=$ 0.4490 (thus a map can be made to the same signal to noise over twice as fast as if we map with all the beams). There is however no tesselation of the unit square into squares of side 0.605 , so the solution is of academic interest only; if we assume instead a value of $p=0.5$ we find that $\mathrm{g}=0.4865$, which is still a saving of over $50 \%$ in the time to make a map. 


\section{APPENDIX 4 Derivation of the aberration function}

In fig.6 the deviation in phase from a spherical wave front at $Q$ for the wave impinging on $P^{\prime}$, a distance $h$ from the telescope axis is equal to $\mathrm{k} \Phi(\mathrm{Q}) . \quad \Phi(\mathrm{Q})$, the aberration function, can be written as an expansion in terms of the coordinates of $Q$ and $\alpha=h / f$, where $f$ is the focal length of the system. For a Cassegrain telescope (i.e. no spherical aberration) with a primary diameter of $\mathrm{D}$ and a subreflector radius of a, $\Phi$ is given in the usual notation by:

$\Phi(\alpha, \hat{\mathbf{r}})=-C \cdot \alpha^{2} \hat{\mathbf{r}}^{2} \cos ^{2} \varphi-\frac{1}{2} D \cdot \alpha^{2} \hat{\mathbf{r}}^{2}+E \cdot \alpha^{3} \hat{\mathbf{r}} \cos \varphi+F \cdot \alpha \hat{\mathbf{r}}^{3} \cos \varphi$

where $\hat{r}=(D / 2 a) . r, r$ being the distance from the centre of the exit pupil of the optical system (the subreflector), and $\varphi$ the angle $r$ makes with the plane determined by the object and the axis of the optical system [15](see fig.5).

The first term (in $C$ ) is the "astigmatism" term

The second (in $D$ ) is the "curvature of field" term.

The third term (in $E$ ) is the "distortion" term and can be neglected for small values of $\alpha$.

The fourth term (in $F$ ) is the "coma" term.

For a Cassegrain where the effective telescope focal length $f$ is much greater than $\mathrm{f}_{\mathrm{p}}$, the primary focal length $C, D$ and $F$ are given by [19]:

$$
C \approx-\gamma \mathrm{d} / 2 \mathrm{fd}_{\mathrm{s}}, \quad D \approx-\mathrm{d} \gamma^{2} / 2 \mathrm{~d}_{\mathrm{s}} \text { and } \quad F=-1 / 4 \mathrm{f}^{2}
$$

where $\gamma=\mathrm{f} / \mathrm{f}_{\mathrm{p}}, \mathrm{d}$ is the distance between the primary and the subreflector and $d_{S}$ is the distance between the subreflector and the Cassegrain focus. For large values of $\gamma, D \gg C$ and so only coma and curvature of field need be considered. Thus,

$$
\Phi(\alpha, r) \approx\left[\gamma h^{2} / 32 F^{3} a\right](r / a)^{2}-\left[h / 32 F^{3}\right](r / a)^{3} \cdot \cos \varphi
$$




\section{APPENDIX 5 Efficiency of an off-axis Gaussian feed}

The efficiency with which the (off-axis) Gaussian feed pattern $E_{g}$ couples to the incoming wave with fields $\mathrm{E}_{\mathrm{S}}$ is given by:

$$
K=\frac{\left.1 \int E_{S} E_{g} d A\right|^{2}}{\int\left|E_{S}\right|^{2} d A \int\left|E_{g}\right|^{2} d A}
$$

where the integration is performed over the plane defined by the subreflector. Thus,

$$
K=\frac{\left.1 \int_{A^{\prime}} \exp \left(-\left(r^{2}+2 h^{\prime} \operatorname{rcos} \varphi-h^{\prime}{ }^{2}\right) / W^{2}\right) \exp \left(j \beta(r / W)^{2}\right) r d r d \varphi\right|^{2}}{\pi W^{2} \cdot A}
$$

where $\beta=\gamma\left(\mathrm{kh} / 32 \mathrm{~F}^{3}\right)(\mathrm{h} / \mathrm{a})(\mathrm{W} / \mathrm{a})^{2}$, i.e. $\beta \mathrm{r}^{2} / \mathrm{W}^{2}=\mathrm{k} \Phi(\mathrm{r})$, and $\mathrm{A}$ is the area of the subreflector.

The integration is performed at the subreflector for values of $r$ from $r$ $=\mathrm{b}$ to $\mathrm{r}=\mathrm{a}$, where $\mathrm{b}$ is the radius of the image of the blocked central region of the primary. First integrating with respect to $\varphi$ gives:

$K=\frac{\left.\mid 2 \pi \int_{A} \cdot I_{0}\left(2 r h / W^{2}\right) \exp \left(-\left(r^{2}-h^{2}{ }^{2}\right) / W^{2}\right) \exp \left(j \beta(r / W)^{2}\right]\right)\left.r d r\right|^{2}}{\pi W^{2} \cdot A}$

where $\mathrm{I}_{\mathrm{O}}$ is the modified Bessel function of order zero.

If the loss in $\mathrm{K}$ is to be small we must have both $\mathrm{h} / \mathrm{W} \ll 1$ and $\beta \ll$ 1. In this case we can write:

and

$$
\begin{aligned}
& I_{0}\left(2 \mathrm{rh} / W^{2}\right) \cdot \exp \left(-(h / W)^{2}\right) \approx 1+(h / W)^{2} \cdot\left[(r / W)^{2}-1\right] \\
& \exp \left(j \beta(\mathrm{r} / W)^{2}\right) \approx 1+j \beta(\mathrm{r} / W)^{2}-\frac{1}{2} \beta^{2}(\mathrm{r} / W)^{2}
\end{aligned}
$$

A change of variable to $\rho=(r / W)^{2}$ gives:

$\mathrm{K} \approx\left(\pi W^{2} / A\right)\left\{\left.1 \int \exp (-\rho) \cdot\left[1+(\mathrm{h} / W)^{2}(\rho-1)-\frac{1}{2} \beta^{2} \rho^{2}\right] \mathrm{d} \rho\right|^{2}\right.$

$$
+1\left\{\left.\hat{\beta} \rho \exp (-\rho) \mathrm{d} \rho\right|^{2}\right\}
$$

where the limits of integration are at $\rho=(b / W)^{2}$ and $(a / W)^{2}$. After some algebra and assuming $b / W \leqslant 1$ we find that:

$\mathrm{K}(\mathrm{h}) \propto\left\{1-2 \cdot g \cdot(\mathrm{h} / \mathrm{a})^{2}-[1-\mathrm{g}(\rho+\mathrm{g})] \cdot \rho^{-2} \cdot\left[\gamma\left(\mathrm{ka} / 32 \mathrm{~F}^{3}\right)\right]^{2}(\mathrm{~h} / \mathrm{a})^{4}\right\}$ 
where $g=\rho \cdot \exp (-\rho) /[1-\exp (-\rho)]$ with $\rho=(a / W)^{2}$.

For a feed with an on-axis nominal taper of $10 \mathrm{~dB}, \rho=1.15$, and $\mathrm{g}=0.5328$, so:

$\mathrm{K}(\mathrm{h}) \propto 1-1.05(\mathrm{~h} / \mathrm{a})^{2}-0.1929\left[\gamma(2 \mathrm{a}) / 16 \lambda \mathrm{F}^{3}\right]^{2}(\mathrm{~h} / \mathrm{a})^{4}$

We express this in terms of the dimensionless parameter $q=h / W_{0}$ where $W_{0}$ is the $1 / \mathrm{e}$ beam radius in amplitude of illuminating feed beam at the phase centre (on the telescope focal plane), using (for a $10 \mathrm{~dB}$ edge taper) $\mathrm{W}_{\mathrm{o}} \approx\left(\lambda \mathrm{d}_{\mathrm{S}} / \pi \mathrm{W}\right)=(0.683 \mathrm{~F} \lambda), \mathrm{d}_{\mathrm{S}}$ is the subreflector-focus distance. Thus:

$K(h) \propto 1-1.95[F \lambda / 2 a]^{2} \cdot q^{2}-2.6 \times 10^{-3} \cdot[\gamma \lambda / F(2 a)]^{2} \cdot q^{4}$

The term that depends on $q^{2}$ is due to the offset, $h$, of the beam from the feed at the subreflector: the term in $q^{4}$ is due to the curvature of field. 


\section{References}

[1] W.N.Christiansen and J.A.Hogböm, Chs.4-7, "Radiotelescopes", Cambridge University Press, 1969.

[2] J.D.Kraus, "Radio Astronomy", Ch.6, McGraw-Hill, 1966.

[3] E.B.Fomalont and M.C.H.Wright, "Interferometery and aperture-synthesis", Ch.10, Galactic and Extra-galactic Radioastronomy", eds.G.L.Verschuur and K.I.Kellermann, Springer-Verlag, 1974

[4] R.Tang and R.W.Burns, "Phased arrays", Ch.20 of "Antenna Engineering Handbook", eds R.C.Johnson and H.Jasik, McGraw-Hill (2nd Edition 1980).

[5] M.Ryle and A.Hewish, "The synthesis of large radiotelescopes", Monthly Not. Roy. Astron. Soc., vol120, pp220-230, 1960.

[6] D.B.Rutledge, D.P.Neikirk and D.P.Kasilingam, "Integrated-circiut antennas", Ch.1 of "Infrared and Millimeter Waves", Vol.10, ed. K.J.Button, Academic Press, 1983.

[7] K.S.Yngvesson, "Near-millimeter wave imaging with integrated planar receptors: general requirements and constraints", Ch.2 of "Infrared and Millimeter Waves", vol.10, ed. K.J.Button, Academic Press, 1983.

[8] J.F.Johansson, K.S.Yngvesson and E.L.Kollberg, "Model experiments with slot antenna arrays for imaging", Instrumentation for Submillimeter Spectroscopy, ed. E.L.Kollberg, Proc. SPIE 598, pp118-125

[9] C.Balanis, "Antenna Theory: Analysis and Design", p.468 Harper \& Row, 1982.

[10] W.N.Christiansen and J.A.Hogböm, p.202, "Radiotelescopes", Cambridge University Press, 1969.

[11] W.C.Wong, "On the equivalent parabola technique to predict the performance characteristics of a Cassegainian system with an off-set feed", IEEE Trans. Antennas Propagat., AP-21, pp335-339, 1973.

[12] P.W.Hannan, "Microwave antennas derived from the Cassegrain telescope", IRE Trans. Antennas Propagat., AP-9, pp140-153, 1961.

[13] J.Ruze, "Lateral-feed displacement in a paraboloid", IEEE Trans., AP-13, pp660-665, 1965. 
[14] H.Gniss and G.Ries, "Remarks on the concept of equivalent parabolas for Cassegrain antennas", Eletron. Letts., vol.6, pp737-739, 1970

[15] M.Born and E.Wolf, "Principles of Optics", Ch.5, Pergammon Press, 1980.

[16] J.A. Murphy, R. Padman and R.E. Hills, "Submillimetre Array Receivers", to be published in Int.J.IR \& Mm Waves, vol.9, no.5. 1988

[17] R.E.Hills in preparation

[18] P.F. Goldsmith, "Quasi-optical techniques at millimeter and submillimeter wavelengths", Ch.5. in "Infrared and Millimeter Waves", ed. K.J. Button, vol.6, Academic Press, 1982

[19] D.Lucas, "Aberrations in Telescopes", in "Advanced Telescope Making Techniques - Vol.1- Optics", Willman-Bell, 1986. 